\title{
Fuzzy Programming Approach to a Multi-Objective Fuzzy Stochastic Routing and Siting Hazardous Wastes
}

\author{
S. Dutta ${ }^{*}$, S. Acharya ${ }^{2}$, Rajashree Mishra ${ }^{2}$ \\ ${ }^{1}$ Department of Mathematics, Assam Don Bosco University, Assam, India. E-mail: duttasanjay098@gmail.com \\ ${ }^{2}$ Department of Mathematics, School of Applied Sciences, KIIT University, Bhubaneswar, India.
}

\begin{abstract}
The aim of the research article is not only to propose a solution procedure to solve multi-objective fuzzy stochastic programming problem by using genetic-algorithm-based fuzzy programming method, but also to apply the computational techniques for transportation of the hazardous waste materials. In this article, routing and siting problems for nuclear hazardous waste material are studied and solved. The amount of waste materials generated in the nuclear reactors follows normal distribution. The two considered objective functions are about route selection which includes minimum travel time and minimum number of houses along the way, taking the safety measures into consideration. A multi-objective fuzzy stochastic mathematical model is formulated with the above mentioned objective functions and the route selection as the constraints. The proposed solution procedure is illustrated by a numerical example and a case study.

Keywords: Fuzzy Stochastic Programming; Multi-Objective Programming; Fuzzy Programming; Genetic Algorithm
\end{abstract}

\section{Introduction}

Hazardous materials and wastes have created a major problem throughout the world. The planning and design of hazardous waste management system involves allocation of hazardous wastes, selection of treatment and disposal facilities, waste residues from generator to the treatment in disposal sites, and selection of the transportation routes. The shipment, dumping of hazardous materials has emerged as a critical problem for decision makers. Some of work done on it can be found in (Ardjmand et al. ${ }^{[1,2]}$, Karadimas et al. ${ }^{[3]}$, Samanlioglu ${ }^{[4]}$, Current and Ratick ${ }^{[5]}$, and Giannikos ${ }^{[6]}$ ). Another issue associated with transporting the hazardous materials is siting problem which may increase the transportation cost if it wasn't considered. The risk factors and the cost associated with transporting the waste materials from source to destination is of great concern for the all the countries which can be seen in (List and Mirchandani ${ }^{[7,8]}$, Nema and Gupta ${ }^{[9]}$, and Gomez et al. ${ }^{[10]}$ ).

These problems are handled with different planning and objectives to meet the common goal of safety and minimization of cost and time. Different methods and approaches are being used and studied to solve the problem in order to make better and accurate decision (Warmerdam and Jacobs ${ }^{[11]}$, Liu ${ }^{[12]}$, Chang and Davi$\left.1 a^{[13]}\right)$. The problem of hazardous wastes treatment demands not only optimal solution but also precise and accurate solution. One of such approach is fuzzy programming approach which is used in various fields to obtain an optimal solution. Some of the applications where the fuzzy programming approaches are used can be found in (Bit et al. ${ }^{[14]}$, Hulsurkar et al. ${ }^{[15]}$, Kumar et al. ${ }^{[16]}$, Liu and $\mathrm{Kao}^{[17]}$ ).

In order to have optimal precise solution, the im-

Copyright (C) 2020 S. Dutta et al.

doi: 10.24294/tm.v3i1.616

EnPress Publisher LLC. This work is licensed under the Creative Commons Attribution-NonCommercial 4.0 International License (CC BY-NC 4.0). http://creativecommons.org/licenses/ by/4.0/ 
preciseness and uncertainty which exist in various parameters need to be handled. This can be done using both the fuzzy theory Zadeh ${ }^{[18]}$ and stochastic programming Charnes and Cooper ${ }^{[19]}$. The idea on the fuzz-ifying approach to multi-objective stochastic programming problem were developed by Mohan and Nguyen ${ }^{[20]}$. Recent developments in fuzzy stochastic problem can be found in (Acharya and Biswal ${ }^{[21]}$, Sakawa et al. ${ }^{[22]}$, Wang and Watada $^{[23]}$, Mousavi et al. ${ }^{[24]}$, Sakawa and Matsui ${ }^{[25]}$, Aiche et al. ${ }^{[26]}$, Acharya et al. ${ }^{[27,28]}$, Li et al. ${ }^{[29]}$ ).

Genetic Algorithm (GA) are based on the concept of the biological process of natural selection, and developed by Holland ${ }^{[30]}$. Liu ${ }^{[12]}$ showed that the existing chance constrained programming models for fuzzy decision systems are essentially maximin model. Also, he analysed that fuzzy simulation based genetic algorithm was suitable for the minimax model. Liu and Iwamura ${ }^{[31]}$ formulated fuzzy simulation based genetic algorithm for solving chance constrained programming models with fuzzy decision. A few researches have been done to handle fuzzy and stochastic using GA (Jana and Biswal ${ }^{[32,33]}$, Dutta et al. ${ }^{[34,35]}$ ).

The article is organized as follows. Following the introduction, basic preliminaries are presented in Section 2. The mathematical model of multi-objective fuzzy stochastic problem is presented describing the alpha-cuts in Section 3. GA based fuzzy programming approach procedure is provided in Section 4. Solution procedure is presented in Section 5. A numerical example and a case study are provided in support of the proposed method in Section 6. Finally, conclusion is provided in Section 7.

\section{Basic preliminaries}

\section{Definition 2.1}

A fuzzy number $\widetilde{A}$ is a convex normalized fuzzy set $\widetilde{A}$ of the real line $\mathfrak{R}$, with membership function $\widetilde{A}$ : $\mathfrak{R} \rightarrow[0,1]$, satisfying the following conditions:

(1) There exist unique interval $J$ such that $\mu_{A}(x)=$ 1: $\mathrm{x} \in \mathrm{J}$

(2) The membership function $\mu_{\mathrm{A}}$ is piecewise continuous.

\section{Definition 2.2}

A fuzzy number $\widetilde{\mathrm{B}}=\left(\mathrm{B}^{(\mathrm{p})}, \mathrm{B}^{(\mathrm{m})}, \mathrm{B}^{(\mathrm{o})}\right)$ is said to be triangular if its membership function is strictly increasing in the interval $\left(\mathrm{B}^{(\mathrm{p})}, \mathrm{B}^{(\mathrm{m})}\right)$ and strictly decreasing in
$\left(\mathrm{B}^{(\mathrm{m})}, \mathrm{B}^{(\mathrm{o})}\right)$ and $\mu_{\mathrm{B}}\left(\mathrm{B}^{(\mathrm{m})}\right)=1$, where $\mathrm{B}(\mathrm{m})$ is core, $\left(\mathrm{B}^{(\mathrm{m})}-\right.$ $\left.B^{(p)}\right)$ is left spread and $\left(B^{(o)}-B^{(m)}\right)$ is right spread of the fuzzy number $\widetilde{\mathrm{B}}^{[36]}$.

\section{Definition 2.3}

$\alpha$ cut of the fuzzy number $\widetilde{\mathrm{A}}$ is the set $\mathrm{x} \mid \mu_{\mathrm{A}}(\mathrm{x})$ $\geq \alpha$ for $0 \leq \alpha<1$ and denoted by $\widetilde{\mathrm{A}}[\alpha]^{[37]}$.

\section{Definition 2.4}

Let $\widetilde{A}=\left(A^{(p)}, A^{(m)}, A^{(o)}\right)$ and $\widetilde{B}=\left(B^{(p)}, B^{(m)}\right.$; $\left.B^{(o)}\right)$ be two fuzzy numbers with $\alpha$ cuts $\widetilde{A}[\alpha]=\left[A_{*}, A^{*}\right]$ and $\widetilde{\mathrm{B}}[\alpha]=\left[\mathrm{B}_{*}, \mathrm{~B}^{*}\right]$ respectively, then $\widetilde{\mathrm{A}} \preccurlyeq \widetilde{\mathrm{B}}$ if and only if $\mathrm{A}_{*} \leq \mathrm{B}_{*}{ }^{[38]}$.

\section{Definition 2.5}

A fuzzy random variable is a random variable whose parameter is fuzzy number. Let $\widetilde{X}$ be continuous random variable with fuzzy parameter $\tilde{\theta}$ and $\widetilde{P}$ as fuzzy probability, then $\widetilde{X}$ is said to be continuous fuzzy random variable with density function $\mathrm{f}(x, \theta)$, $\tilde{P}(\tilde{X} \leq \mathrm{x})=\beta$, where $0 \leq \beta \leq 1 ; \tilde{\beta}=\left(\beta^{(p)}, \beta^{(m)}\right.$, $\left.\beta^{(o)}\right), \beta^{(o)} \geq 0$ and $\beta^{(\mathrm{p})} \geq 0^{[39]}$.

\section{Definition 2.6}

Let $E=[c, d]$ be an event. Then the probability of the event $\mathrm{E}$ of continuous fuzzy random variable $\widetilde{\mathrm{X}}$ is a fuzzy number whose $\alpha$ cut is ${ }^{[39]}$

$\widetilde{\mathrm{P}}[\mathrm{c} \leq \widetilde{\mathrm{X}} \leq \mathrm{d}]=$ $\left[\min \left\{\int_{\mathrm{a}}^{\mathrm{b}} \mathrm{f}(\mathrm{x}, \theta) \mathrm{dx} \mid \theta \in \widetilde{\theta}[\alpha] ; \int_{-\infty}^{\infty} \mathrm{f}(\mathrm{x}, \theta)=1\right\}\right.$ $\left.\max \left\{\int_{\mathrm{a}}^{\mathrm{b}} \mathrm{f}(\mathrm{x}, \theta) \mathrm{dx} \mid \theta \in \widetilde{\theta}[\alpha] ; \int_{-\infty}^{\infty} \mathrm{f}(\mathrm{x}, \theta)=1\right\}\right]$ $=\left[\beta_{*}[\alpha], \beta^{*}[\alpha]\right]$

Bounded Random Number (BRN): For any type of $\mathrm{C}$ compiler, the subfunction of generating pseudo random number has been given in the $\mathrm{C}$ library as: include < stdlib.h > and rand function rand () which produces an integer between 0 and RAND MAX, where RAND MAX is in \# stdlib as $\left(2^{15}-1\right)$. Therefore, a random number on an interval $[0,1]$ can be generated as:

Step 1: $\mathrm{m}=$ rand ()

Step 2: $\mathrm{m} \leftarrow(\mathrm{m}=\mathrm{RAND}$ MAX $)$

Fuzzy Normal Distribution: A random variables has a fuzzy normal distribution if its probability density function (pdf) is given by:

$$
\mathrm{f}(\mathrm{s})=\frac{1}{\widetilde{\sigma} \sqrt{2 \pi}} \mathrm{e}^{\left\{-\frac{(\mathrm{s}-\widetilde{\mu})^{2}}{2 \widetilde{\sigma}^{2}}\right\}},-\infty<\mathrm{s}<\infty
$$

Denote the pdf as $\overparen{F N L}\left(\tilde{\mu}, \widetilde{\sigma}^{2}\right)$, where $\tilde{\mu}$ is the mean and $\widetilde{\sigma}^{2}$ is the variance

Step 1: Generate $n_{1}$ and $n_{2}$ from $\operatorname{BRN}(0,1)$

Step 2: $\mathrm{m}=\left[-2 \ln \left(\mathrm{n}_{1}\right)\right]^{0.5} \operatorname{Sin}\left(2 \pi \mathrm{n}_{2}\right)$ 
Step 3: Return $(\tilde{\mu}+\tilde{\sigma}$ m)

Fuzzy Exponential Distribution: A random variables has a fuzzy exponential distribution if it probability density function (pdf) is defined as

$f(s)=\left\{\begin{array}{l}\frac{1}{\tilde{\beta}} \mathrm{e}^{-\frac{s}{\tilde{\beta}}}, \text { if } s>0 \\ 0, \quad \text { otherwise }\end{array}\right.$

Denote the pdf as $\widetilde{\operatorname{FEXP}}(\widetilde{\beta})$, where $\tilde{\beta}$ is the mean. It can be generated as follows

Step 1: Generates from BRN $(0,1)$

Step 2: Return $-\widetilde{\beta} \ln (\mathrm{s})$

Fuzzy Weibull Distribution: A random variables has a fuzzy Weibull distribution if its probability density function (pdf) is defined as

$f(s)=\left\{\begin{array}{c}\frac{\tilde{\beta}}{\tilde{\gamma}^{\widetilde{\beta}}} s^{\widetilde{\beta}-1} \mathrm{e}^{(\widetilde{\tilde{\gamma}})^{\widetilde{\beta}}}, \text { if } s>0 \\ 0, \text { otherwise }\end{array}\right.$

Denote the pdf as $\overline{F W}(\tilde{\beta}, \tilde{\gamma})$, where $\tilde{\beta}$ is the shape parameter and $\tilde{\gamma}$ is the scale parameter, and $\tilde{\beta}>$ $0 ; \tilde{\gamma}>0$. It can be generated as follows

Step 1: Generates from EXP (1)

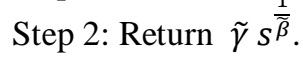

\section{Mathematical model of mul- ti-objective fuzzy stochastic pro- gramming problem}

The mathematical programming model for multi-objective fuzzy stochastic programming (MOFSP) problem is expressed as

$\min Z_{k}=\sum_{j=1}^{n} C_{j}^{k} x_{j}, k=1,2, \ldots \ldots K$

Subject to

$\widetilde{P}\left(\sum_{j=1}^{n} a_{i j} x_{j} \preccurlyeq \widetilde{b}_{l}\right) \geqslant\left(1-\widetilde{\beta}_{l}\right), i=$

$1,2, \ldots, m$

$x_{j} \geq 0, \forall j$

where $\beta_{i}$ is the fuzzy number and $C_{j}^{k} \in \mathfrak{R}, \forall j, k$

Depending on the different distribution of the random variables, following cases are considered in this article.

Case 1: Let $b_{i}, i=1,2, \ldots, m$ are independent FRVs distributed normally. Let the FRVs $b_{i}$ be denoted as $\widetilde{b}_{l}$.

The $\alpha$-cut of the probabilistic constrained defined above can be expressed as:

$\widetilde{P}\left(\sum_{j=1}^{n} a_{i j} x_{j} \preccurlyeq \widetilde{b}_{l}\right)[\alpha], i=1,2, \ldots, m$

$=\left\{P\left(A_{i} \leq b_{i}\right) \mid b_{i} \in \widetilde{b}_{l}[\alpha]\right\}$, where $A_{i}=$

$\sum_{j=1}^{n} a_{i j} x_{\mathrm{j}}$

Using fuzzy inequality, the $\alpha$-cut of the fuzzy constraints $(3.4-3.5)$ is expressed as:

$\widetilde{P}\left(\sum_{j=1}^{n} a_{i j} x_{j} \leqslant \widetilde{b_{l}}\right)[\alpha] \geqslant\left(1-\beta_{i}[\alpha]\right)=\left\{P\left(A_{i} \leq\right.\right.$

$\left.\left.b_{i *}\right) \geq\left(1-\beta_{i}^{*}\right)\right\}$

where

$\left[b_{i *}[\alpha], b_{i}^{*}[\alpha]\right] \in b_{i}[\alpha]$ and $\left[\beta_{i *}[\alpha], \beta_{i}^{*}[\alpha]\right] \in \beta_{i}[\alpha]$

Case 2: Let $b_{i}, i=1,2, \ldots, m$ are independent FRVs distributed Weibull. Let the FRVs $b_{i}$ be denoted as $\widetilde{b}_{l}$.

The $\alpha$-cut of the probabilistic constrained defined above can be expressed as:

$\widetilde{P}\left(\sum_{j=1}^{n} a_{i j} x_{j} \preccurlyeq \widetilde{b_{l}}\right)[\alpha], i$

$=1,2, \ldots, m$

$=\left\{P\left(A_{i} \leq b_{i}\right) \mid b_{i} \in \widetilde{b}_{l}[\alpha]\right\}$, where $A_{i}$

$$
=\sum_{j=1}^{n} a_{i j} x_{j}
$$

Using fuzzy inequality, the $\alpha$-cut of the fuzzy constraints $(3.7-3.8)$ is expressed as:

$$
\begin{aligned}
& \widetilde{P}\left(\sum_{j=1}^{n} a_{i j} x_{j} \preccurlyeq \widetilde{b}_{l}\right)[\alpha] \\
& \geqslant\left(1-\beta_{i}[\alpha]\right) \\
& =\left\{P\left(A_{i} \leq b_{i *}\right)\right. \\
& \left.\geq\left(1-\beta_{i}^{*}\right)\right\} \\
& \quad \text { where } \\
& {\left[b_{i *}[\alpha], b_{i}^{*}[\alpha]\right] \in b_{i}[\alpha] \text { and }\left[\beta_{i *}[\alpha], \beta_{i}^{*}[\alpha]\right] \in \beta_{i}[\alpha]}
\end{aligned}
$$

\section{Fuzzy programming approach simulation based GA}

The fuzzy programming simulation-based GA is design to solve the fuzzy probabilistic programming problems. A fuzzy stochastic simulation-based GA algorithm is described in Dutta et al. ${ }^{[35]}$. We describe the steps of the algorithm as follows: 


\section{end while}

$x_{i n}^{*}$

// Ideal Solution for each objective function separately \}

End

The Flow Diagram of Fuzzy Stochastic GA is shown in Figure 1.

\subsection{Representation and initialization}

A population of potential solution $x_{1}, x_{2}, \ldots \ldots, x_{n}$ is generated and initialized. Each solution is called chromosomes can be represented as $X_{p}=$ $\left(x_{1}, x_{2}, \ldots \ldots, x_{n}\right)_{p}$, where $\mathrm{p}=1,2, \ldots \ldots, \mathrm{p} \_$size and p_size being the size of the population.

\subsection{Checking constraints by the fuzzy simu- lation}

The constraints of the model are represented as fuzzy probabilistic constraints. Consider the fuzzy probabilistic constraints

$\widetilde{P}\left(\sum_{j=1}^{n} a_{i j} x_{j} \leq \widetilde{b}_{l}\right)[\alpha] \geqslant\left(1-\beta_{i}[\alpha]\right)$,

$\mathrm{i}=1,2, \ldots \ldots, \mathrm{m}$

We defuzzifying the constraints using the $\alpha$-cut and inequality conditions, so that the constraint reduces to $\left\{P\left(A_{i} \leq b_{i *}\right) \geqslant\left(1-\beta_{i}^{*}\right)\right\}, j=1,2, \ldots \ldots, n$ as discuss before

The above inequality can be represented by

$$
\begin{aligned}
\left\{P\left(A_{i} \leq b_{i *}\right) \geq\right. & \left.\left(1-\beta_{i}^{*}\right)\right\}, j \\
& =1,2, \ldots ., n \\
=P\left(s_{i}\left(a_{i j} x_{j}, s_{1}\right)\right. & \leq 0) \geq\left(1-\beta_{i}^{*}\right), j \\
& =1,2, \ldots, n
\end{aligned}
$$

where $s_{1}=b_{i *}, i=1,2, \ldots, m ; x_{j}=\left(x_{1}, x_{2}, \ldots \ldots, x_{n}\right)$ is the decision variables.

Let $N_{i j}(\leq N), i=1,2, \ldots, m ; j=1,2, \ldots \ldots, n$ be the number of times the following relation are satisfied: $s_{i}\left(a_{i j} x_{j}\right) \leq 0, i=1,2, \ldots, m ; j=1,2, \ldots . ., n$

And $s_{j}\left(a_{i j} x_{j}\right) \geq 0, i=1,2, \ldots, m ; j=1,2, \ldots ., n$, where $N$ is the initial population. Then, by the definition of probability, (3.2) hold, if $\frac{N_{i j}}{N} \geq\left(1-\beta_{i}\right), i=$ $1,2 \ldots \ldots, m ; j=1,2, \ldots ., n$.

\subsection{Fitness}

The value of objective function is called fitness value if the given constraints are satisfied. 


\subsection{Selection}

Selection operator selects the best chromosomes depending on the fitness values. In this article, we choose Binary Tournament Selection (BTS) for selection process.

\subsection{Crossover}

Probability of crossover (pc) is assigned, and a random number is generated within $[0,1]$ for each pair of chromosomes. If the random number is less than (pc), then the chromosomes are selected for mating.

\subsection{Mutation}

Probability of mutation (pm) is assigned, and a random number is generated within $(0,1)$ for each chromosome. If the random number is less than (pm), the chromosome will undergo mutation.

\subsection{Termination}

When number of iterations becomes equal to the generation number as defined, the execution will be stopped.

\section{Solution procedure}

Using GA based fuzzy programming approach, we find out the ideal solution for each objective function in the following way:

Step 1: Initialize the population $P_{1}(\mathrm{t}), P_{2}(\mathrm{t}), \ldots$,
$P_{n}(\mathrm{t})$, for each objective function $\left(Z_{1}(\mathrm{x}), Z_{2}(\mathrm{x})\right.$,

$\left.Z_{n}(\mathrm{x})\right)$ separately, keeping the constraints same for all the objective functions.

Step 2: Apply fuzzy stochastic GA based approach to find the ideal solution for each objective function. (See Figure 1)

Step 3: Construct a pay-off matrix containing the ideal solution and functional values.

Step 4: From the constructed pay-off matrix, determine the bounds for $\mathrm{m}$-th objective function $Z_{m}(x) ; \mathrm{m}=$ $1,2, \ldots \ldots, \mathrm{n}$. As there are only two possible choices for the bound, either a lower bound or an upper bound. Find the best lower bound $L_{m}^{* *}$ and worst upper bound $U_{m}^{*}$ in case an objective function being of minimization type and in case of maximization type finding the best upper bound $U_{m}^{* *}$ and worst lower bound $L_{m}^{*} ; m=$ $1,2, \ldots \ldots, n$.

Step 5: Define fuzzy membership function $\mu_{z m}$ for the m-th objective function $Z_{m}(x)$ as:

$$
\left\{\begin{array}{c}
1, \quad \text { if } Z_{m}(x) \geq U_{m}^{* *}, \quad m=1,2, \ldots, n \\
\frac{Z_{m}(\mathrm{x})-L_{m}^{*}}{U_{m}^{* *}-L_{m}^{*}}, \text { if } L_{m}^{*}<Z_{m}(x)<U_{m}^{* *} \\
0, \quad \text { if } Z_{m}(x) \leq L_{m}^{*}
\end{array}\right.
$$

$$
\text { Or }
$$

$$
\left\{\begin{array}{c}
1, \quad \text { if } Z_{m}(x) \geq L_{m}^{* *}, \quad m=1,2, \ldots, n \\
\frac{U_{m}^{*}-Z_{m}(x)}{U_{m}^{*}-L_{m}^{* *}}, \text { if } L_{m}^{* *}<Z_{m}(x)<U_{m}^{*} \\
0, \quad \text { if } Z_{m}(x) \geq U_{m}^{* *}
\end{array}\right.
$$

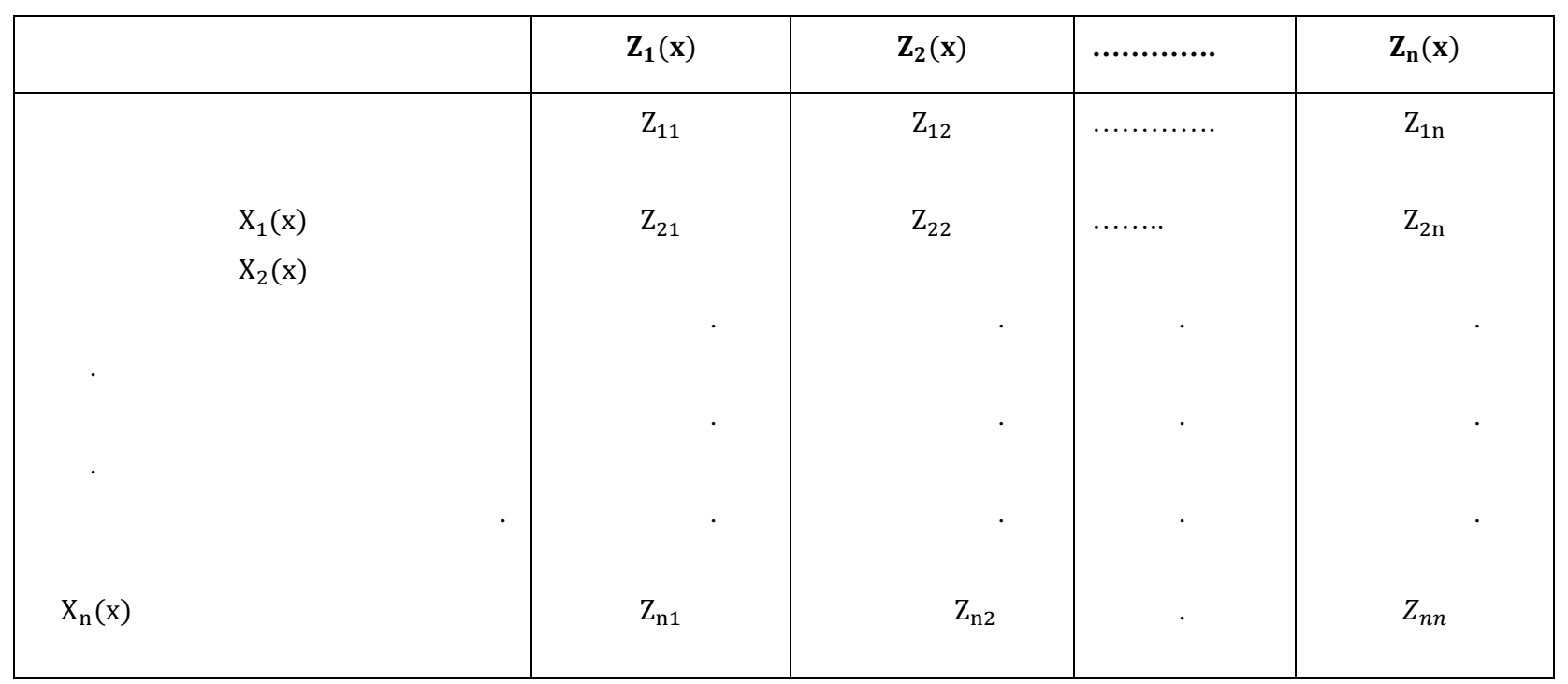

Table 1. Pay-off matrix 
Step 6: (i) Formulate a single objective mathematical programming problem using maxmin operator with augmented variable $\lambda$

$\max : \lambda$

Subject to

$\lambda \leq \mu z_{p}(x), p=1,2,3, \ldots \ldots . R$

$x \in S$

where $\mathrm{S}$ is the feasible region of the MOFSP model.

(ii) Similarly, using min-max operator with augmented variable $\lambda$, and formulating a single objective mathematics programming problem as:

$\min : \lambda$

Subject to

$\lambda \geq \mu z_{p}(x), \mathrm{p}=1,2,3, \ldots \ldots . R$

$x \in S$

where $S$ is the feasible region of the MOFSP model.

Step 7: Finally, solve the single objective mathematical programming problem (5.3) - (5.5) or (5.6) - (5.8) using GA-based fuzzy stochastic approach to obtain the Pareto solutions.

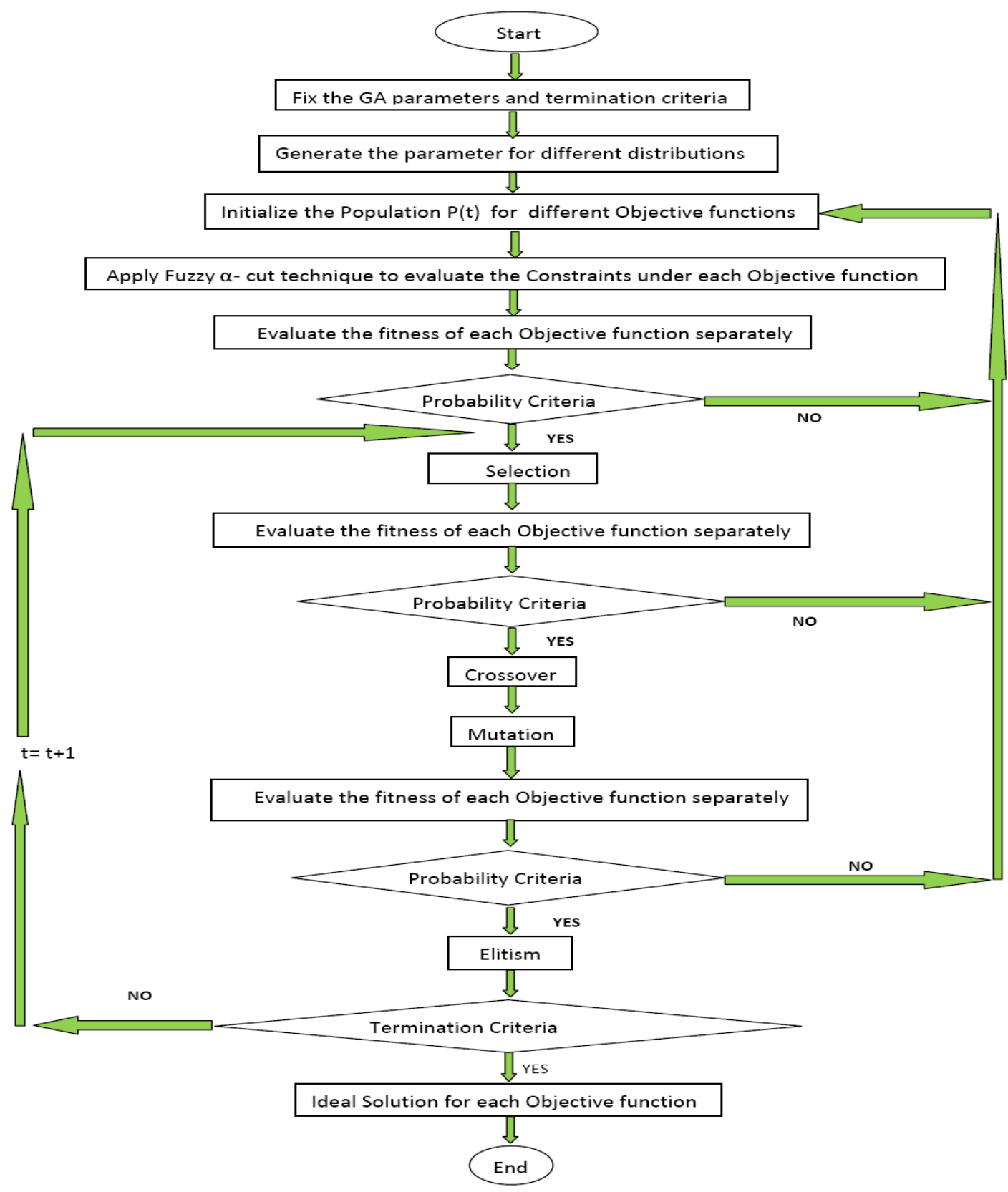

Figure 1. Flow diagram of fuzzy stochastic fuzzy programming approach GA. 


\section{Numerical example}

Consider the following example:

$$
\begin{aligned}
& \max =5 x_{1}+7 x_{2} \\
& \max =10 x_{1}-2 x_{2} \\
& \max =9 x_{1}+8 x_{2} \\
& \quad \text { Subject to } \\
& \widetilde{P}\left(2 x_{1}+x_{2} \leq \widetilde{b_{1}}\right) \geqslant \widetilde{0.7} \\
& \widetilde{P}\left(x_{1}+2 x_{2} \leq \widetilde{b_{2}}\right) \geqslant \widetilde{0.60} \\
& 2 x_{1}+2 x_{2} \leq 4 \\
& x_{1}, x_{2}>0
\end{aligned}
$$

where $\widetilde{b_{1}}$ and $\widetilde{b_{2}}$ follows Weibull distribution with $\widetilde{F W}\left(\widetilde{\beta_{b_{1}}}, \widetilde{\gamma_{b_{1}}}\right)=\widetilde{F W}(\tilde{4}, \tilde{2}) \quad, \quad \widetilde{F W}\left(\widetilde{\beta_{b_{2}}}, \widetilde{\gamma_{b_{2}}}\right)=$ $\widetilde{F W}(\tilde{5}, \tilde{2}) \quad$ where $\tilde{2}=(2 / 3 / 4) ; \tilde{4}=(4 / 5 / 6) ; \tilde{5}=$ $(5 / 6 / 7)$ are fuzzy triangular numbers respectively.

After applying the steps of fuzzy programming approach, we obtain three ideal solutions as $X_{1}(x)=$ (0.90458; 1.08882); $Z_{1}(x)=12.1446 ; \quad X_{2}(x)=$ (1.09666; 0.06076); $Z_{2}(x)=10.8451 ;$ and, $X_{3}(x)=$ $(1.0709 ; 0.9253) ; Z_{3}(x)=17.0405$.

\begin{tabular}{|l|l|l|l|}
\hline & \multicolumn{1}{|c|}{$\mathrm{Z}_{1}$} & \multicolumn{1}{|c|}{$\mathrm{Z}_{2}$} & \multicolumn{1}{c|}{$\mathrm{Z}_{3}$} \\
\hline $\mathrm{X}_{1}$ & 12.1446 & 6.86816 & 16.85178 \\
\hline $\mathrm{X}_{2}$ & 5.90832 & 10.8451 & 1.35602 \\
\hline $\mathrm{X}_{3}$ & 11.8361 & 8.8584 & 17.0405 \\
\hline
\end{tabular}

Table 2. The pay-off matrix

Use max-min operator with augmented $\lambda$ the $\tilde{P}\left(x_{1}+2 x_{2} \leq \widetilde{b_{2}}\right)$ MOFSP reduces to MOFSGP problem $\min : \lambda$

$$
\geq \widetilde{0.60}
$$

\begin{tabular}{|c|c|c|c|c|c|c|}
\hline pc & pm & Optimum Solution $X=\left(x_{1}, x_{2}\right)$ & $\mathbf{Z}=\left(\mathbf{Z}_{1}, \mathbf{Z}\right.$ & $\left(2, Z_{3}\right)$ & & $\lambda$ \\
\hline 0.6 & 0.01 & $1.0481,0.4921$ & 8.6852 & 9.4968 & 13.3697 & 0.9 \\
\hline 0.6 & 0.03 & $1.0614, \quad 0.5415$ & 9.0975 & 9.531 & 13.8846 & 0.5 \\
\hline 0.6 & 0.05 & $1.05855,0.7448$ & $\begin{array}{l}10.5064 \\
15.4854\end{array}$ & & 9.0959 & 0.9 \\
\hline 0.6 & 0.08 & $1.0481, \quad 0.60515$ & $\begin{array}{l}9.47655 \\
14.2741\end{array}$ & & 9.2707 & 0.9 \\
\hline 0.7 & 0.01 & $1.06045, \quad 0.8702$ & $\begin{array}{l}11.3937, \\
16.5056\end{array}$ & & 8.8641 & 0.5 \\
\hline 0.7 & 0.03 & $1.0519, \quad 0.76$ & 10.5795 & 8.999 & 15.5471 & 0.5 \\
\hline 0.7 & 0.05 & $1.0405, \quad 0.88255$ & $\begin{array}{l}11.3804 \\
16.4249\end{array}$ & & 8.6399 & 0.6 \\
\hline 0.7 & 0.08 & $1.06995, \quad 0.68685$ & $\begin{array}{l}10.1577 \\
15.1244\end{array}$ & & 9.3258 & 0.9 \\
\hline 0.8 & 0.01 & $1.03385, \quad 0.6099$ & $\begin{array}{l}9.43855 \\
14.1839 \\
\end{array}$ & & 9.1187 & 0.8 \\
\hline 0.8 & 0.03 & $1.06805, \quad 0.4522$ & 8.50565 & 9.7761, & 13.23 & 0.8 \\
\hline 0.8 & 0.05 & $1.05285, \quad 0.84265$ & $\begin{array}{l}\text { 11.1628, } \\
16.2169\end{array}$ & & 8.8432 & 0.6 \\
\hline 0.8 & 0.08 & $1.0614, \quad 0.89395$ & 11.5647 & & 8.8261, & 0.6 \\
\hline
\end{tabular}

$$
\begin{aligned}
& 2 x_{1}+2 x_{2} \leq 4 \\
& x_{1}, x_{2}>0
\end{aligned}
$$
(6.9) The proposed GA based fuzzy programming ap-
(6.10) proach is coded in $\mathrm{C}++$ in VB2010 professional. The (6.11) population size is taken as 100 . Optimum solution ob(6.12) tained for different values of probability of crossover, mutation and $\alpha=0.5$ over 100 generation is presented. 


\begin{tabular}{|c|c|c|c|c|c|c|c|}
\hline & & & & 16.7042 & & & \\
\hline 0.9 & 0.01 & 1.06805, & 0.31825 & $\begin{array}{l}7.568, \\
12.1584\end{array}$ & & 10.044 & 0.8 \\
\hline 0.9 & 0.03 & 1.06235, & 0.79135 & 10.8512 , & 9.0408 , & 15.892 & 0.5 \\
\hline 0.9 & 0.05 & 1.06425, & 0.3515 & $\begin{array}{l}7.78175, \\
12.3903\end{array}$ & & 9.9395 & 0.9 \\
\hline 0.9 & 0.08 & 1.0367, & 0.7391 & $\begin{array}{l}10.3572, \\
15.2431\end{array}$ & & 8.8888, & 0.6 \\
\hline
\end{tabular}

Table 3. $\alpha=0.5$

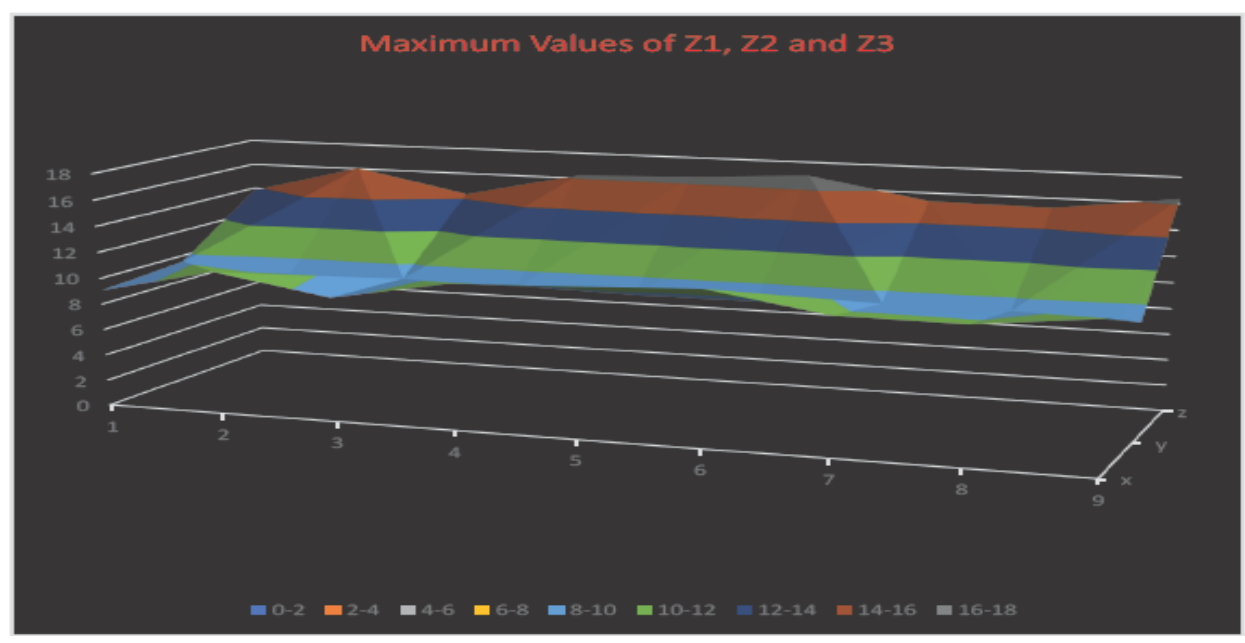

Figure 2. Maximum values of $\mathrm{Z}_{1}, \mathrm{Z}_{2}$ and $\mathrm{Z}_{3}$

\subsection{Case study}

The need for the energy in the world is kept on increasing, and one of the major sources of energy is nuclear power. As a result of using nuclear as a fuel to generate energy, the waste products generated in the reactor needs to be handled and disposed very carefully, as they are hazardous to the environment and mankind. To dispose the nuclear waste in different sites away from the reactor requires shipment across a transportation network, which has become one of the major problems. To transport the nuclear waste material from the reactor to different sites, one has to look into several factors, such as environment issues, accident prone area, time taken to reach the sites, distance covered from reactor to different sites, population along the road or rail through which waste will be transported, and cost of transportation.

\begin{tabular}{|l|l|l|l|l|l|}
\hline Source & & Route 1/Site 1 & Route 1/Site 2 & Route 2/Site 1 & Route 2/Site 2 \\
\hline 1 & Time(hours) & 5 & 7 & 13 & 18 \\
\hline 2 & No. of Houses & 900 & 400 & 3000 & 2000 \\
\hline & Time(hours) & 8 & 9 & 12 & 15 \\
\hline
\end{tabular}




\begin{tabular}{|l|l|l|l|l|l|}
\hline & No. of Houses & 1800 & 800 & 2500 & 1000 \\
\hline 3 & Time(hours) & 6 & 18 & 10 & 13 \\
\hline & No. of Houses & 3000 & 1700 & 1500 & 800 \\
\hline
\end{tabular}

Table 4. Model information

The mathematical model of the case study can be expressed as follows:

$$
\begin{array}{r}
\text { Min: } Z_{1}=5 x_{111}+7 x_{112}+13 x_{121}+18 x_{122}+8 x_{211}+ \\
9 x_{212}+12 x_{221}+15 x_{222}+16 x_{311}+18 x_{312}+ \\
10 x_{321}+13 x_{322}
\end{array}
$$

Min: $Z_{2}=900 x_{111}+400 x_{112}+3000 x_{121}+$

$$
\begin{array}{r}
2000 x_{122}+1800 x_{212}+2500 x_{221}+1000 x_{222}+ \\
3000 x_{311}+1700 x_{312}+1500 x_{321}+800 x_{322}
\end{array}
$$

Subject to

$$
\begin{aligned}
& \tilde{P}\left(x_{111}+x_{112}+x_{121}+x_{122} \preccurlyeq \widetilde{b_{1}}\right) \geqslant \widetilde{0.75} \\
& \tilde{P}\left(x_{211}+x_{212}+x_{221}+x_{222} \preccurlyeq \widetilde{b_{2}}\right) \geqslant \widetilde{0.85} \\
& \tilde{P}\left(x_{311}+x_{312}+x_{321}+x_{322} \preccurlyeq \widetilde{b_{3}}\right) \geqslant \widetilde{0.70}
\end{aligned}
$$

$$
\sum_{j=1}^{2} y_{j}=1
$$$$
x_{i j k} \leq b_{i} y_{j}, i=1,2,3 ; j=1,2 ; k=1,2
$$$$
y_{j}=0 \text { or } 1, j=1,2
$$

where $b_{1}, b_{2}$, and $b_{3}$ follows normal distribution with $\widetilde{F N L}\left(\widetilde{\mu_{b_{1}}}, \widetilde{\sigma_{b_{1}}^{2}}\right)=\widetilde{F N L}(\tilde{3}, \tilde{1}) \quad, \quad \widetilde{F N L}\left(\widetilde{\mu_{b_{2}}}, \widetilde{\sigma_{b_{2}}^{2}}\right)=$ $\widetilde{F N L}(\tilde{2}, \tilde{1}), \widetilde{F N L}\left(\widetilde{\mu_{b_{3}}}, \widetilde{\sigma_{b_{3}}^{2}}\right)=\widetilde{F N L}(\tilde{4}, \tilde{2})$ respectively, where $\tilde{1}=(1,2,3), \quad \tilde{2}=(2,3,4), \tilde{3}=(3,4,5), \tilde{4}=$ $(4,5,6)$ are the fuzzy triangular numbers.

Applying the steps of fuzzy programming approach, we obtain two ideal solutions as $X_{1}(x)=0.7118,0.2644$, $0.1126,0.1655,0.5541,0.5946,0.2976,0.2007,0.1143$, (6.18) $\quad 0.1507,0.1468,0.1689, Z_{1}(x)=34.4201$, and $X_{2}(x)=$ $0.69915,0.1851,0.146,0.25295,0.2938,0.1342,0.3204$, $0.37265, \quad 0.2157, \quad 0.1442, \quad 0.1962, \quad 0.217, \quad Z_{2}(x)=$ 4817.165 .

\begin{tabular}{|c|c|c|}
\hline & \multicolumn{1}{|c|}{$\mathbf{Z}_{1}$} & \multicolumn{1}{|c|}{$\mathbf{Z}_{2}$} \\
\hline$c$ & 34.4201 & 6914.185 \\
\hline $\mathrm{X}_{2}$ & 35.0651 & 4817.165 \\
\hline
\end{tabular}

Table 5. The pay-off matrix

Using min-max operator with augmented $\lambda$ the MOFSP reduces to MOFSGP

$\min : \lambda$

Subject to

$$
\begin{aligned}
& 5 x_{111}+7_{112}+13 x_{121}+18 x_{122}+8 x_{211}+9 x_{212}+ \\
& 12 x_{221}+15 x_{222}+16 x_{311}+18 x_{312}+10 x_{321}+ \\
& 13 x_{322}+0.645 \lambda \geq 35.065
\end{aligned}
$$

$900 x_{111}+400 x_{112}+3000 x_{121}+2000 x_{122}+$ $1800 x_{212}+2500 x_{221}+1000 x_{222}+3000 x_{311}+$ $1700 x_{312}+1500 x_{321}+800 x_{322}+2097 \lambda 6914.185$

$\tilde{P}\left(x_{111}+x_{112}+x_{121}+x_{122} \preccurlyeq \widetilde{b_{1}}\right) \geqslant \widetilde{0.75}$

$$
\begin{aligned}
& \tilde{P}\left(x_{211}+x_{212}+x_{221}+x_{222} \preccurlyeq \widetilde{b_{2}}\right) \geqslant \widetilde{0.85} \\
& \tilde{P}\left(x_{311}+x_{312}+x_{321}+x_{322} \preccurlyeq \widetilde{b_{3}}\right) \geqslant \widetilde{0.70} \\
& \sum_{j=1}^{2} y_{j}=1 \\
& x_{i j k} \leq b_{i} y_{j}, i=1,2,3 ; j=1,2 ; k=1,2 \\
& y_{j}=0 \text { or } 1, j=1,2
\end{aligned}
$$

The proposed GA based fuzzy programming approach is coded in $\mathrm{C}++$ in VB2010 professional. The population size is taken as 100 . Optimum solution Table (6-14) obtained for different values of probability of crossover (pc), mutation (pm) and $\alpha$ over 100 generations are presented. 


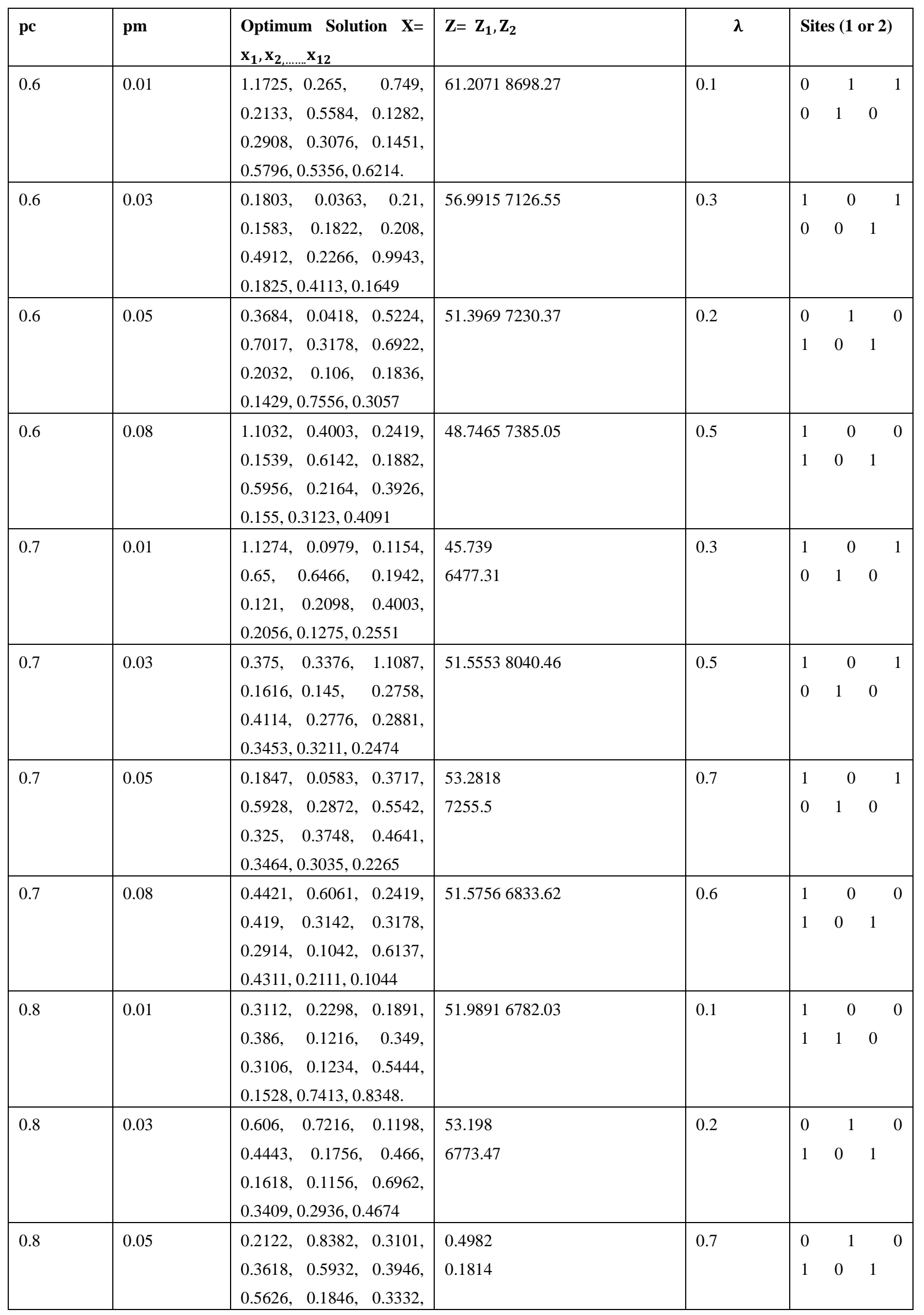




\begin{tabular}{|c|c|c|c|c|c|c|c|}
\hline & & $0.2045,0.4982,0.1814$ & & & & & \\
\hline 0.8 & 0.08 & $\begin{array}{lll}0.7677, & 0.253, & 0.1473, \\
0.3156, & 0.2698, & 0.2914, \\
0.5416, & 0.1486, & 0.2232, \\
0.2771, & 0.1649, & 0.43\end{array}$ & 47.24365823 .34 & 0.7 & $\begin{array}{l}0 \\
0\end{array}$ & $\begin{array}{llll} & 1 & & \\
& & & \\
1 & & 0 & \end{array}$ & \\
\hline 0.9 & 0.01 & $\begin{array}{lll}0.7952, & 0.1716, & 0.7149, \\
0.2221, & 0.5002, & 0.3946, \\
0.2548, & 0.1312, & 0.1077, \\
0.2804, & 0.1638, & 0.5752\end{array}$ & $\begin{array}{l}46.9333 \\
6863.1\end{array}$ & 0.9 & $\begin{array}{l}0 \\
1\end{array}$ & 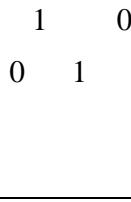 & \\
\hline 0.9 & 0.03 & $\begin{array}{lll}0.2859, & 0.5246, & 0.1165, \\
0.3739, & 0.6892, & 0.5686, \\
0.3502, & 0.652, & 0.3981, \\
0.1451, & 0.1374, & 0.6269\end{array}$ & 56.46496935 .98 & 0 & $\begin{array}{l}0 \\
0\end{array}$ & 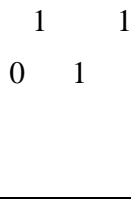 & \\
\hline 0.9 & 0.05 & $\begin{array}{lll}0.5785, & 0.2321, & 0.3277, \\
0.2958, & 0.2692 & 0.3334, \\
0.2584, & 0.436, & 0.1847, \\
0.2628, & 0.7677, & 0.2243\end{array}$ & 47.54336353 .69 & 0.6 & 0 & $\begin{array}{llll} & 0 & & 1 \\
& & & \\
1 & & 0\end{array}$ & \\
\hline 0.9 & 0.08 & $\begin{array}{lll}0.3959, & 0.3883, & 0.1616, \\
0.1836, & 0.3688, & 0.1174, \\
0.5776, & 0.6406, & 0.3387, \\
0.3431, & 0.5323, & 0.2386\end{array}$ & 50.67026794 .69 & 0.3 & $\begin{array}{l}0 \\
1\end{array}$ & 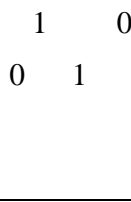 & \\
\hline
\end{tabular}

Table 6. $\alpha=0.1$

\begin{tabular}{|c|c|c|c|c|c|c|c|}
\hline pc & pm & Optimum Solution $\mathrm{X}=$ & $\mathrm{Z}=\mathrm{Z}_{1}, \mathrm{Z}_{2}$ & $\lambda$ & \multicolumn{3}{|c|}{ Sites (1 or 2$)$} \\
\hline 0.6 & 0.01 & $\begin{array}{lcc}0.9558, & 0.066, & 0.1374, \\
0.2089, & 0.349, & 0.2542, \\
0.154, & 0.61, & 0.1814, \\
0.5466, & 0.672, & 0.3211\end{array}$ & 50.50076281 .48 & 0.5 & $\begin{array}{l}1 \\
0\end{array}$ & $\begin{array}{l}0 \\
1\end{array}$ & $\begin{array}{ll} & 1 \\
0 & \end{array}$ \\
\hline 0.6 & 0.03 & $\begin{array}{lll}0.573, & 0.0099, & 0.397, \\
0.1506, & 0.5812, & 0.5002, \\
0.5626, & 0.1558, & 0.1099, \\
0.1671, & 0.9613, & 0.3145\end{array}$ & 52.60067332 .89 & 0.2 & $\begin{array}{l}1 \\
0\end{array}$ & $\begin{array}{l}0 \\
1\end{array}$ & $\begin{array}{rr} & 1 \\
0 & \end{array}$ \\
\hline 0.6 & 0.05 & $\begin{array}{lll}0.1913, & 0.0264, & 0.21, \\
0.1154, & 0.505, & 0.2236, \\
0.6946, & 0.2134, & 0.3013, \\
0.2892, & 0.1352, & 0.8029\end{array}$ & 45.35326321 .97 & 0.3 & $\begin{array}{l}0 \\
1\end{array}$ & $0^{1}$ & $\begin{array}{l}0 \\
1\end{array}$ \\
\hline 0.6 & 0.08 & $\begin{array}{lll}0.4454, & 0.4378, & 0.1154, \\
0.3937, & 0.2008, & 0.2686, \\
0.1462, & 0.1576, & 0.1869, \\
1.1186, & 0.4905, & 0.3156\end{array}$ & 54.15366259 .55 & 0.8 & $\begin{array}{l}0 \\
1\end{array}$ & $0^{1}$ & $1^{0}{ }^{0}$ \\
\hline 0.7 & 0.01 & $\begin{array}{lll}0.2782, & 1.1417, & 0.1968, \\
0.2463, & 0.1504, & 0.3052, \\
0.1528, & 0.2932, & 0.5312, \\
0.4322, & 0.3013,1.1351\end{array}$ & 60.60446668 .51 & 0.6 & & $\begin{array}{l}0 \\
0\end{array}$ & $\begin{array}{l}0 \\
1\end{array}$ \\
\hline
\end{tabular}




\begin{tabular}{|c|c|c|c|c|c|c|c|}
\hline 0.7 & 0.03 & $\begin{array}{lll}0.848, & 0.1683, & 0.1077, \\
0.9503, & 0.5326, & 0.4894, \\
0.1852, & 0.2566, & 0.1363, \\
0.1066, & 0.2749, & 0.3222\end{array}$ & 49.69766384 .25 & 0.4 & $\begin{array}{l}1 \\
0\end{array}$ & $\begin{array}{l}0 \\
1\end{array}$ & $0^{1}$ \\
\hline 0.7 & 0.05 & $\begin{array}{lll}0.2837, & 0.0055, & 0.8359, \\
0.1066, & 0.6358, & 0.373, \\
0.2764, & 0.1654, & 0.2254, \\
0.1011, & 0.6874, & 0.3926\end{array}$ & 45.88777470 .92 & 0.1 & $\begin{array}{l}0 \\
1\end{array}$ & $0^{1}$ & $1^{0}$ \\
\hline 0.7 & 0.08 & $\begin{array}{lll}0.7512, & 0.4411, & 0.2155, \\
0.144, & 0.2356, & 0.1822, \\
0.433, & 0.172, & 0.2188, \\
0.5521, & 0.3222, & 0.2254\end{array}$ & 56.46215883 .28 & 0.5 & $\begin{array}{l}1 \\
0\end{array}$ & $\begin{array}{l}0 \\
0\end{array}$ & $\begin{array}{l}1 \\
1\end{array}$ \\
\hline 0.8 & 0.01 & $\begin{array}{lll}0.32, & 0.3894, & 0.4322, \\
0.1539, & 0.3574, & 0.3796, \\
0.2962, & 0.3502, & 0.7644, \\
0.2177, & 0.2628, & 0.1176\end{array}$ & 48.10347237 .43 & 0.7 & $\begin{array}{l}1 \\
0\end{array}$ & $\begin{array}{l}0 \\
1\end{array}$ & $0^{1}$ \\
\hline 0.8 & 0.03 & $\begin{array}{lll}0.8777, & 0.0011, & 0.1583, \\
0.1616, & 0.5572, & 0.3028, \\
0.1954, & 0.6142, & 0.2837, \\
0.1847, & 0.4069, & 0.2958\end{array}$ & 47.92825952 .5 & 0.6 & $\begin{array}{l}1 \\
0\end{array}$ & $\begin{array}{l}{ }^{0} \\
0\end{array}$ & $1^{1}$ \\
\hline 0.8 & 0.05 & $\begin{array}{lll}0.1902, & 0.5225, & 0.1473, \\
0.2892, & 0.1846, & 0.2122, \\
0.5488, & 0.6772, & 0.2045, \\
0.3343, & 1.1285, & 0.1044\end{array}$ & 53.79086909 .8 & 0.3 & $\begin{array}{l}1 \\
0\end{array}$ & $\begin{array}{l}0 \\
1\end{array}$ & $0^{1}$ \\
\hline 0.8 & 0.08 & $\begin{array}{lll}0.5565, & 0.3564, & 0.3871, \\
0.1022, & 0.2038, & 0.2746, \\
0.4528, & 0.1144, & 0.3112, \\
0.4003, & 1.0449, & 0.1022\end{array}$ & 50.71337108 .6 & 0.6 & $\begin{array}{l}1 \\
1\end{array}$ & $\begin{array}{l}0 \\
0\end{array}$ & $\begin{array}{l}0 \\
1\end{array}$ \\
\hline 0.9 & 0.01 & $\begin{array}{lll}0.3277, & 0.1936, & 0.1209, \\
0.2254, & 0.7024, & 0.5158, \\
0.5968, & 0.2488, & 0.3937 \\
0.595, & 0.2177, & 0.3849\end{array}$ & 56.07047432 .8 & 0.5 & $\begin{array}{l}1 \\
0\end{array}$ & $\begin{array}{l}0 \\
1\end{array}$ & $\begin{array}{l}1 \\
0\end{array}$ \\
\hline 0.9 & 0.03 & $\begin{array}{lll}0.2837, & 0.8745, & 0.265, \\
0.177, & 0.1564, & 0.3082, \\
0.1, & 0.2638, & 0.5202, \\
0.7127, & 0.1847, & 0.5026\end{array}$ & 52.88566247 .33 & 0.7 & $\begin{array}{l}1 \\
0\end{array}$ & $\begin{array}{l}0 \\
1\end{array}$ & $0^{1}$ \\
\hline 0.9 & 0.05 & $\begin{array}{lll}0.9514, & 0.3817, & 0.1737, \\
0.3101, & 0.313 & 0.4342, \\
0.2746, & 0.517, & 0.3794, \\
0.177, & 0.8678, & 0.1231 \\
\end{array}$ & 53.66957105 .18 & 0.2 & $\begin{array}{l}1 \\
0\end{array}$ & $\begin{array}{l}0 \\
1\end{array}$ & $\begin{array}{l}1 \\
0\end{array}$ \\
\hline 0.9 & 0.08 & $\begin{array}{llc}0.3739, & 0.4113, & 0.2386, \\
0.1649, & 0.3334, & 0.3706, \\
0.3658, & 0.1936, & 0.133, \\
0.2199, & 0.6137,0.386\end{array}$ & 46.0825558 .24 & 0.7 & $\begin{array}{l}0 \\
0 \\
1\end{array}$ & 1 & $\begin{array}{l}1 \\
0\end{array}$ \\
\hline
\end{tabular}

Table 7. $\alpha=0.2$ 


\begin{tabular}{|c|c|c|c|c|c|c|c|c|c|c|}
\hline pc & pm & Optimum Solution $X=x_{1}, x_{2, \ldots \ldots . . .} x_{12}$ & $\mathbf{Z}=\mathrm{Z}_{1}, \mathrm{Z}_{2}$ & $\lambda$ & \multicolumn{6}{|c|}{ Sites (1 or 2$)$} \\
\hline 0.6 & 0.01 & $\begin{array}{l}0.1308,0.4455,0.1253,0.1132,0.208,0.184 \\
0.2836,0.1582,0.9448,0.3156,0.5015,0.1099\end{array}$ & 43.77656498 .11 & 0.8 & 1 & 0 & 1 & 0 & 1 & 0 \\
\hline 0.6 & 0.03 & $\begin{array}{l}0.3464,0.088,0.6808,0.2166,0.3298,0.2014 \\
0.1288,0.3688,0.2155,0.6566,0.32,0.6291\end{array}$ & 53.27097014 .12 & 0 & 0 & 1 & 0 & 1 & 0 & 1 \\
\hline 0.6 & 0.05 & $\begin{array}{l}1.0526,0.0858,0.2254,0.1198,0.1012,0.5056 \\
0.3382,0.1726,0.2155,1.1593,0.2848,0.2716\end{array}$ & 56.01416766 .35 & 0.2 & 1 & 0 & 1 & 0 & 1 & 0 \\
\hline 0.6 & 0.08 & $\begin{array}{l}0.3321,0.0968,0.8942,0.2078,0.4282,0.3472 \\
0.1642,0.2644,0.3651,0.5092,0.3508,0.1319\end{array}$ & 50.41987751 .89 & 0.2 & 0 & 1 & 0 & 1 & 0 & 1 \\
\hline 0.7 & 0.01 & $\begin{array}{l}0.177,0.5357,0.3046,0.1363,0.586,0.1804 \\
0.2488,0.2254,0.2309,0.7479,0.5158,0.5334\end{array}$ & 52.97516771 .05 & 0.7 & 1 & 0 & 1 & 0 & 1 & 0 \\
\hline 0.7 & 0.03 & $\begin{array}{l}0.6599,0.4378,0.2012,0.3739,0.5782,0.3754 \\
0.2986,0.2128,0.2606,0.5114,0.2694,0.1077\end{array}$ & 47.95826562 .25 & 0.2 & 1 & 0 & 1 & 0 & 1 & 0 \\
\hline 0.7 & 0.05 & $\begin{array}{l}0.3992,0.1782,0.188,0.3376,0.1768,0.1882 \\
0.6106,0.4648,0.6027,0.2001,0.1946,0.188\end{array}$ & 55.42986729 .05 & 0.3 & 0 & 1 & 0 & 1 & 0 & 1 \\
\hline 0.7 & 0.08 & $\begin{array}{l}0.2672,0.5158,0.4806,0.155, \quad 0.6814,0.4438 \\
0.409,0.1288,0.3849,0.2012,0.1418,0.6049\end{array}$ & 49.33157124 .82 & 0.7 & 0 & 1 & 0 & 1 & 1 & 0 \\
\hline 0.8 & 0.01 & $\begin{array}{l}0.4245,0.0561,1.0834,0.1253,0.1954,0.2038 \\
0.112,0.538, \quad 0.3178,0.3607,0.1594,0.7336\end{array}$ & 54.37447630 .62 & 0.2 & 0 & 1 & 0 & 1 & 0 & 1 \\
\hline 0.8 & 0.03 & $\begin{array}{l}0.6412,0.5665,0.6995,0.177, \quad 0.1102,0.4582 \\
0.4102,0.1522,0.4454,0.5301,0.1088,0.3057\end{array}$ & 53.39217643 .93 & 0.1 & 0 & 1 & 0 & 1 & 0 & 1 \\
\hline 0.8 & 0.05 & $\begin{array}{l}0.947,0.4301,0.1176,0.2056,0.2404,0.1582 \\
0.4258,0.1276,0.2804,0.2045,0.6225,0.3541\end{array}$ & 45.14575948 .4 & 0.8 & 1 & 0 & 0 & 1 & 0 & 1 \\
\hline 0.8 & 0.08 & $\begin{array}{l}0.8018,0.8338,0.5015,0.6258,0.3118,0.2992 \\
0.637,0.1378,0.1517,0.1297,0.2452,0.1011\end{array}$ & 51.05587466 .41 & 0 & 0 & 1 & 0 & 1 & 0 & 1 \\
\hline 0.9 & 0.01 & $\begin{array}{l}0.661,1.0043,0.1891,0.2518,0.5632,0.4132 \\
0.3334,0.6826,0.2441,0.1319,0.122,0.859\end{array}$ & 58.45686754 .67 & 0.3 & 0 & 1 & 0 & 1 & 0 & 1 \\
\hline 0.9 & 0.03 & $\begin{array}{l}0.9327,0.9394,0.1737,0.419, \quad 0.4468,0.4942, \\
0.2392,0.2662,0.3035,0.3002,0.1154,0.2188\end{array}$ & 50.1836407 .07 & 0.8 & 1 & 0 & 1 & 0 & 1 & 0 \\
\hline 0.9 & 0.05 & $\begin{array}{ll}0.5598,0.1903,0.3959,0.1407, & 0.4108,0.7006 \\
0.2332,0.5506,0.1022,0.353, & 0.2936,0.771\end{array}$ & 53.40786446 .46 & 0.6 & 1 & 0 & 1 & 0 & 1 & 0 \\
\hline 0.9 & 0.08 & $\begin{array}{l}0.6786,0.6193,0.331,0.1792,0.3754,0.1924 \\
0.2362,0.1144,0.2837,0.6214,0.8293,0.7226\end{array}$ & 57.95317473 .91 & 0.1 & 1 & 0 & 1 & 0 & 1 & 0 \\
\hline
\end{tabular}

Table 8. $\alpha=0.3$

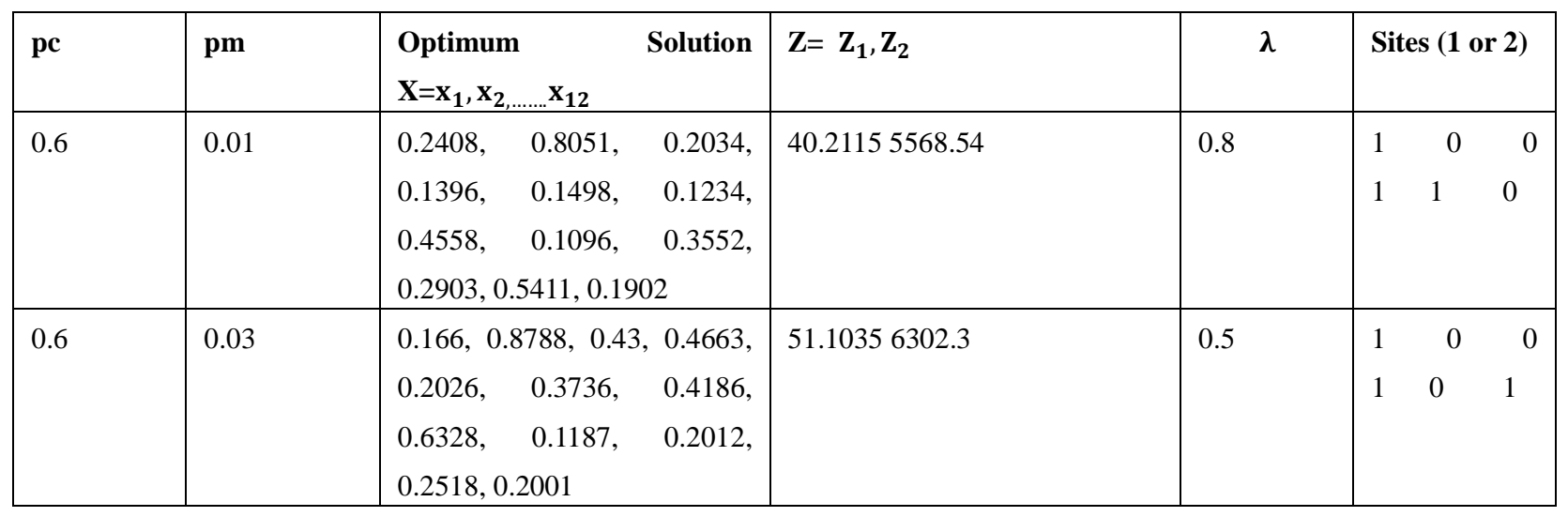




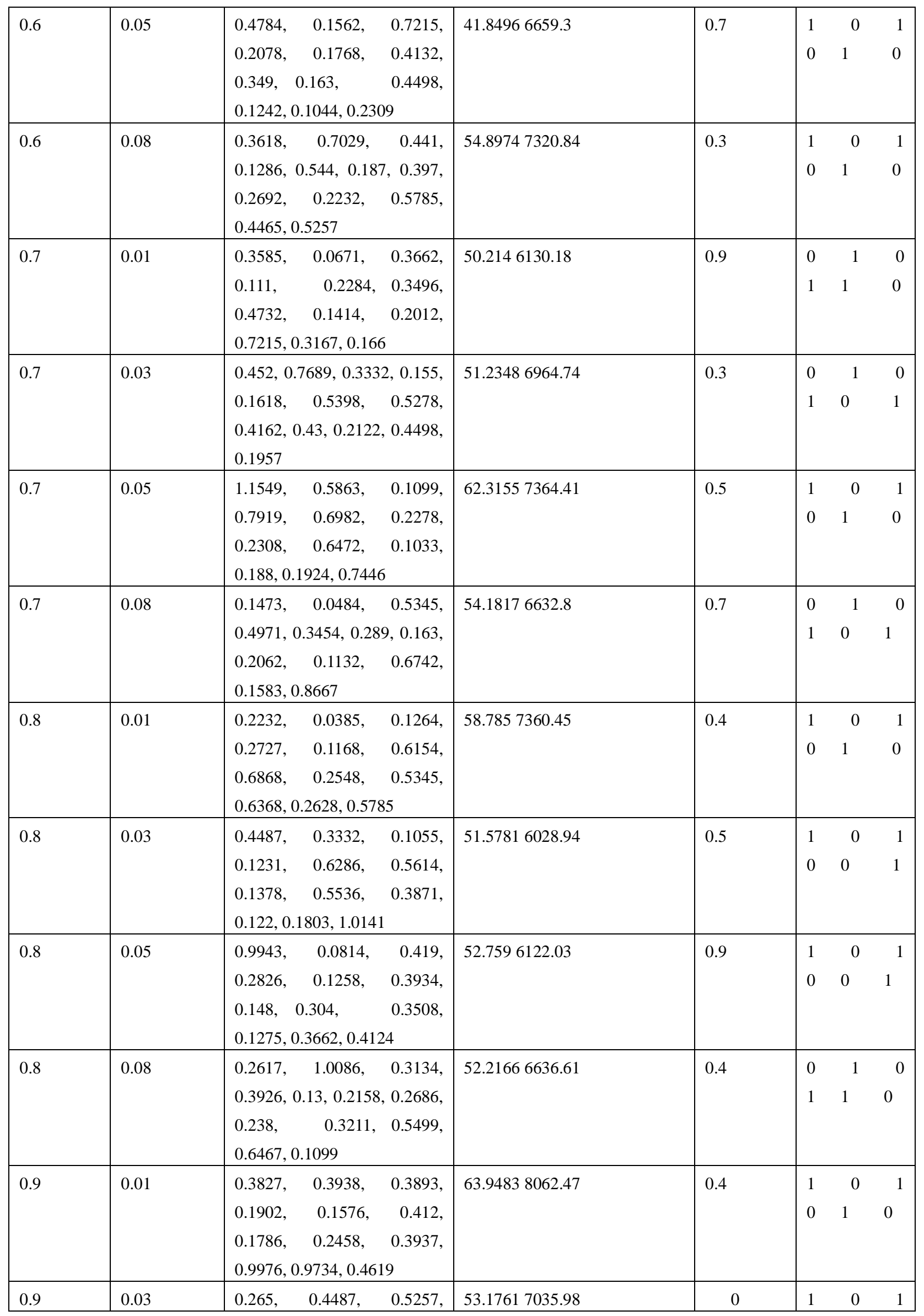




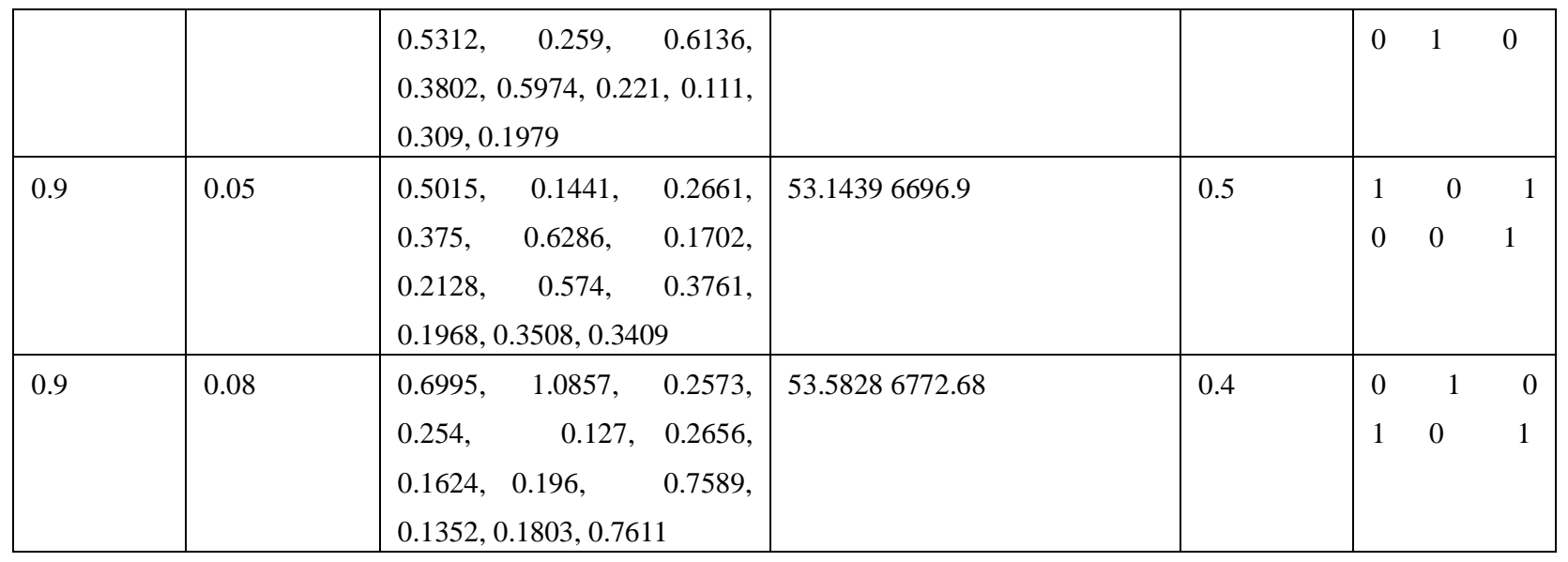

Table 9. $\alpha=0.4$

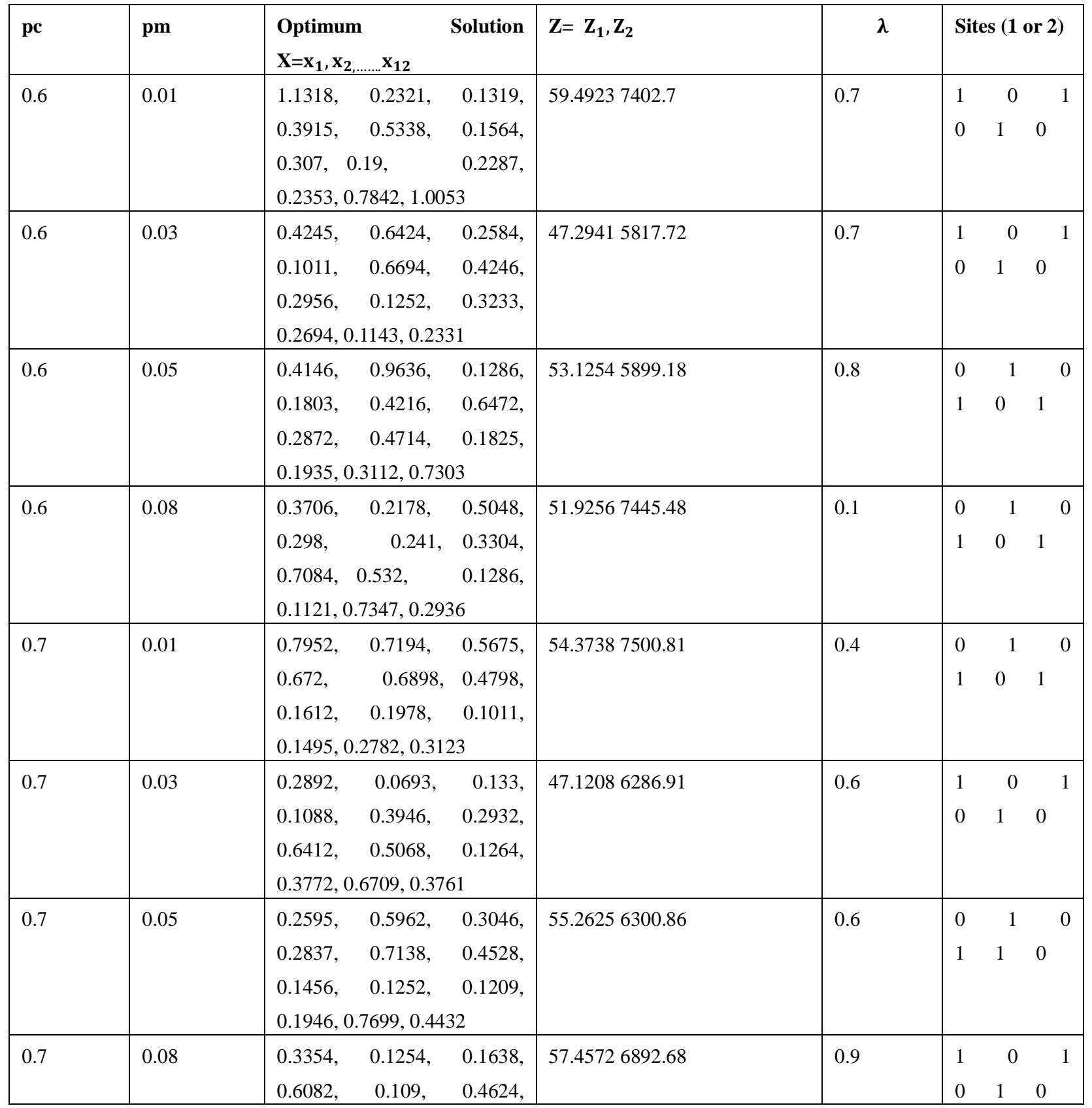




\begin{tabular}{|c|c|c|c|c|c|c|c|}
\hline & & $\begin{array}{l}0.2788, \quad 0.1942, \quad 0.7028, \\
0.1407,0.5015,0.3354\end{array}$ & & & & & \\
\hline 0.8 & 0.01 & $\begin{array}{lll}0.6665, & 0.4235, & 0.1374, \\
0.5004, & 0.2554, & 0.2902, \\
0.1042, & 0.226, & 0.1495, \\
0.2133, & 0.8084,0.6698\end{array}$ & 58.33715929 .11 & 0.9 & $\begin{array}{l}1 \\
0\end{array}$ & $\begin{array}{lll} & 0 & \\
1 & 0\end{array}$ & \\
\hline 0.8 & 0.03 & $\begin{array}{lll}0.3695, & 0.6358, & 0.3827, \\
0.2155, & 0.1996, & 0.5146, \\
0.2794, & 0.1816, & 0.7897, \\
0.3134, & 0.1176,0.6621\end{array}$ & 55.68037425 .15 & 0.1 & $\begin{array}{l}0 \\
1\end{array}$ & $\begin{array}{lll} & 1 & \\
0 & 1\end{array}$ & \\
\hline 0.8 & 0.05 & $\begin{array}{lll}0.7853, & 0.0154, & 0.5136, \\
0.1341, & 0.3328, & 0.3484, \\
0.2248, & 0.1372, & 0.5994, \\
0.4168, & 0.1517,0.4608\end{array}$ & 60.28077213 .84 & 0.1 & $\begin{array}{l}1 \\
1\end{array}$ & $\begin{array}{lll} & 0 & \\
0 & 1\end{array}$ & \\
\hline 0.8 & 0.08 & 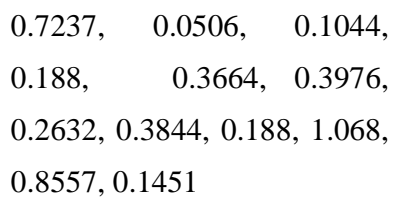 & 56.82327160 & 0.5 & $\begin{array}{l}1 \\
0\end{array}$ & $\begin{array}{lll} & 0 & \\
1 & 0\end{array}$ & \\
\hline 0.9 & 0.01 & $\begin{array}{l}0.2815, \quad 0.2914, \quad 0.1671, \\
0.4278, \quad 0.244, \quad 0.1354, \\
0.1816,0.7036,0.7523,0.54, \\
0.4003,0.2177\end{array}$ & 57.81377381 .44 & 0.5 & $\begin{array}{l}0 \\
0\end{array}$ & $\begin{array}{ll}1 & 1 \\
0 & 1\end{array}$ & \\
\hline 0.9 & 0.03 & $\begin{array}{lll}0.7006, & 1.0142, & 0.4773, \\
0.3651, & 0.1636, & 0.5434, \\
0.6652, & 0.3274, & 0.1814, \\
0.1231, & 0.1022,0.2584\end{array}$ & 51.97137031 .41 & 0.1 & $\begin{array}{l}0 \\
1\end{array}$ & $\begin{array}{lll} & 1 & \\
0 & 1\end{array}$ & \\
\hline 0.9 & 0.05 & $\begin{array}{lcc}0.518, & 0.0462, & 0.2375, \\
0.6621, & 0.13, & 0.2296, \\
0.178, & 0.1366, & 0.3981, \\
0.5917, & 0.2166,0.3871\end{array}$ & 49.42866355 .43 & 0.7 & $\begin{array}{l}0 \\
1\end{array}$ & $\begin{array}{lll} & & \\
0 & 1 & \end{array}$ & \\
\hline 0.9 & 0.08 & $\begin{array}{lll}0.3585, & 0.7084, & 0.5961, \\
0.4036, & 0.1684, & 0.2668, \\
0.1336, & 0.1366, & 0.3596, \\
0.3552, & 0.5235,0.4377\end{array}$ & 56.31987010 .8 & 0.9 & $\begin{array}{l}0 \\
0\end{array}$ & $\begin{array}{lll} & 1 & \\
1 & 0\end{array}$ & \\
\hline
\end{tabular}

Table 10. $\alpha=0.5$

\begin{tabular}{|c|c|c|c|c|c|c|c|c|c|}
\hline pc & pm & $\begin{array}{l}\text { Optimum } \\
\mathrm{X}=\mathrm{x}_{\mathbf{1}}, \mathrm{x}_{2, \ldots \ldots} \mathrm{x}_{12}\end{array}$ & Solution & $\mathbf{Z}=\mathbf{Z}_{1}, \mathbf{Z}_{2}$ & $\lambda$ & \multicolumn{4}{|c|}{ Sites (1 or 2$)$} \\
\hline 0.6 & 0.01 & $\begin{array}{ll}0.1319, & 1.0263 \\
0.3068, & 0.2806 \\
0.3022, & 0.1792 \\
0.5444, & 0.2903,0\end{array}$ & $\begin{array}{r}0.1847, \\
0.1318, \\
0.1319, \\
55\end{array}$ & 44.78285259 .41 & 0.8 & $\begin{array}{l}1 \\
0\end{array}$ & $\begin{array}{l}0 \\
1\end{array}$ & & 1 \\
\hline 0.6 & 0.03 & $\begin{array}{lr}0.188, & 0.2475, \\
0.1066, & 0.1378 \\
0.286, & 0.2566, \\
0.9096, & 0.2287,0 .\end{array}$ & $\begin{array}{l}0.2859, \\
0.4846, \\
0.5807, \\
6\end{array}$ & 55.45186974 .69 & 0.5 & & $\begin{array}{l}0 \\
1\end{array}$ & & 1 \\
\hline
\end{tabular}




\begin{tabular}{|c|c|c|c|c|c|c|c|}
\hline 0.6 & 0.05 & $\begin{array}{lcc}0.3607, & 0.6611, & 0.2606, \\
0.4674, & 0.1666, & 0.2878, \\
0.2248, & 0.1012, & 0.144, \\
0.7567, & 0.7446,0.8282\end{array}$ & 60.5086996 .84 & 0 & $\begin{array}{l}0 \\
1\end{array}$ & $0^{1}$ & $\begin{array}{r}0 \\
1\end{array}$ \\
\hline 0.6 & 0.08 & 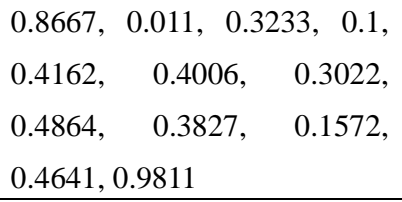 & 54.61897162 .24 & 0.8 & $\begin{array}{l}0 \\
1\end{array}$ & $0^{1}$ & $\begin{array}{l}0 \\
1\end{array}$ \\
\hline 0.7 & 0.01 & $\begin{array}{lll}0.914, & 0.297,0.4025, & 0.3871, \\
0.5044, & 0.2836, & 0.1384, \\
0.1696, & 0.2793, & 0.2056, \\
0.2606,0.3277 & \end{array}$ & 44.67746413 .98 & 0.6 & $\begin{array}{l}0 \\
1\end{array}$ & $0^{1}$ & $1^{0}$ \\
\hline 0.7 & 0.03 & $\begin{array}{lll}0.3805, & 0.8084, & 0.1451,0.32, \\
0.1756, & 0.3976, & 0.1288, \\
0.4372, & 0.4179, & 0.1242, \\
0.4212, & 0.2881 & \\
\end{array}$ & 45.17375461 .59 & 0.9 & $\begin{array}{l}0 \\
0\end{array}$ & $\begin{array}{l}{ }^{1} \\
1\end{array}$ & \\
\hline 0.7 & 0.05 & $\begin{array}{lcc}0.2166, & 0.4147, & 0.4201, \\
0.1066, & 0.1606, & 0.277, \\
0.1978, & 0.3556, & 0.199, \\
1.2132, & 0.2551,0.3849\end{array}$ & 55.42776545 .11 & 0.2 & $\begin{array}{l}1 \\
0\end{array}$ & $\begin{array}{l}0 \\
1\end{array}$ & \\
\hline 0.7 & 0.08 & $\begin{array}{lcc}0.2529, & 0.5939, & 0.2529, \\
0.287, & 0.4558, & 0.5848, \\
0.226, & 0.3148, & 0.5565, \\
0.1484, & 0.3992,0.4256\end{array}$ & 51.31916827 .01 & 0.3 & $\begin{array}{l}0 \\
1\end{array}$ & $\begin{array}{l}{ }^{1} \\
1\end{array}$ & $0^{0}$ \\
\hline 0.8 & 0.01 & $\begin{array}{lll}0.1528, & 0.6281, & 0.5257, \\
0.5004, & 0.5308, & 0.5116, \\
0.2782, & 0.643, & 0.1913, \\
0.1814, & 0.1891,0.1055\end{array}$ & 52.42476920 .21 & 0.3 & $\begin{array}{l}1 \\
0\end{array}$ & $\begin{array}{l}0 \\
1\end{array}$ & $0^{1}$ \\
\hline 0.8 & 0.03 & $\begin{array}{lcc}0.2023, & 0.5082, & 0.2507, \\
0.2529, & 0.271, & 0.3982, \\
0.1552, & 0.2548, & 0.5917, \\
0.1836, & 0.4168,0.3189\end{array}$ & 51.49456066 .54 & 0.9 & $\begin{array}{l}0 \\
1\end{array}$ & $\begin{array}{l}{ }^{1} \\
1\end{array}$ & $\begin{array}{c}0 \\
0\end{array}$ \\
\hline 0.8 & 0.05 & $\begin{array}{lcc}0.3486, & 0.2199, & 0.2584, \\
0.5477, & 0.148, & 0.4234, \\
0.3634, & 0.6172, & 0.1495, \\
0.3288, & 0.4333,0.6632\end{array}$ & 56.37856591 .09 & 0.5 & $\begin{array}{l}1 \\
0\end{array}$ & $\begin{array}{l}{ }^{0} \\
0\end{array}$ & $\begin{array}{l}1 \\
1\end{array}$ \\
\hline 0.8 & 0.08 & $\begin{array}{lcc}0.4091, & 0.2475, & 0.3673, \\
0.1726, & 0.2218, & 0.478, \\
0.3784, & 0.1702, & 0.7105, \\
0.2001,0.1517,0.4036\end{array}$ & 48.58556836 .25 & 0.1 & $\begin{array}{l}0 \\
1\end{array}$ & $0^{1}$ & $\begin{array}{l}0 \\
1\end{array}$ \\
\hline 0.9 & 0.01 & $\begin{array}{lll}0.8898, & 0.0781, & 0.1957, \\
0.6148, & 0.3442, & 0.3304, \\
0.124, & 0.3832, & 0.7138, \\
0.1638, & 0.2254,0.4234\end{array}$ & 53.69687322 .52 & 0.2 & $\begin{array}{l}0 \\
1\end{array}$ & $0^{1}$ & $1^{0}$ \\
\hline
\end{tabular}




\begin{tabular}{|c|c|c|c|c|c|c|c|c|}
\hline 0.9 & 0.03 & $\begin{array}{lr}0.6863, & 0.731 \\
0.3431, & 0.262,0 \\
0.5176, & 0.404 \\
0.2562, & 0.3783\end{array}$ & $\begin{array}{l}0.3607 \\
0.2716 \\
0.1814\end{array}$ & 52.73476820 .95 & & $\begin{array}{l}0 \\
1\end{array}$ & $0^{1}$ & $\begin{array}{l}0 \\
1\end{array}$ \\
\hline 0.9 & 0.05 & $\begin{array}{lr}1.0691, & 0.191 \\
0.2023, & 0.156 \\
0.4474, & 0.244 \\
0.2463, & 0.2595,0 \\
\end{array}$ & $\begin{array}{l}0.1132, \\
0.1576, \\
0.6819, \\
9\end{array}$ & 54.2976636 .51 & 0.4 & 1 & $\begin{array}{l}0 \\
0\end{array}$ & $\begin{array}{l}0 \\
1\end{array}$ \\
\hline 0.9 & 0.08 & $\begin{array}{cr}0.4322, & 0.38 \\
0.1176, & 0.578 \\
0.2896, & 0.658 \\
0.276,0.9833,0 .\end{array}$ & $\begin{array}{l}0.4014, \\
0.3412, \\
0.1396,\end{array}$ & 51.86927139 .65 & 0.4 & $\begin{array}{l}0 \\
1\end{array}$ & $\begin{array}{l}1 \\
1\end{array}$ & $\begin{array}{l}0 \\
0\end{array}$ \\
\hline
\end{tabular}

Table 11. $\alpha=0.6$

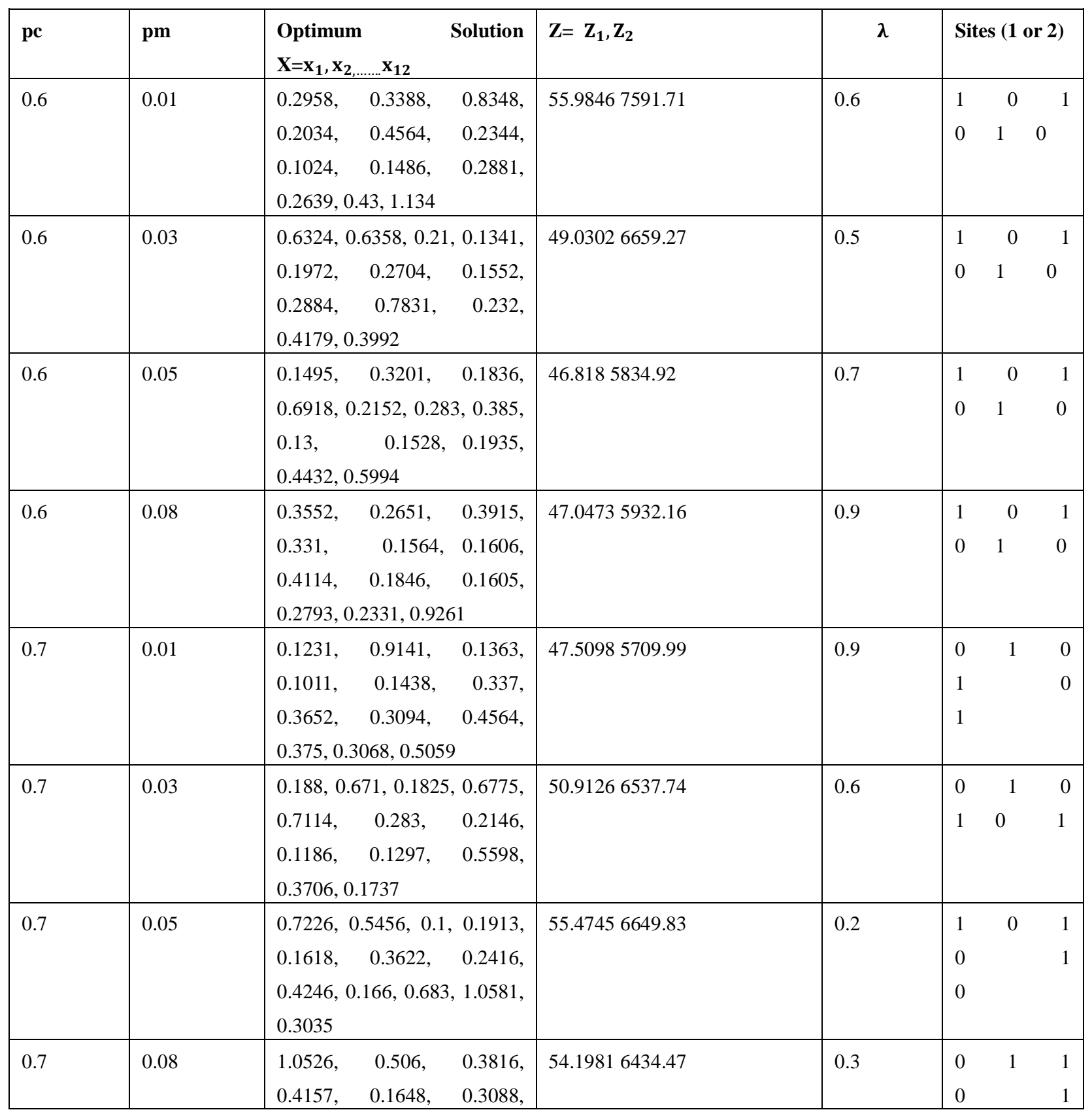




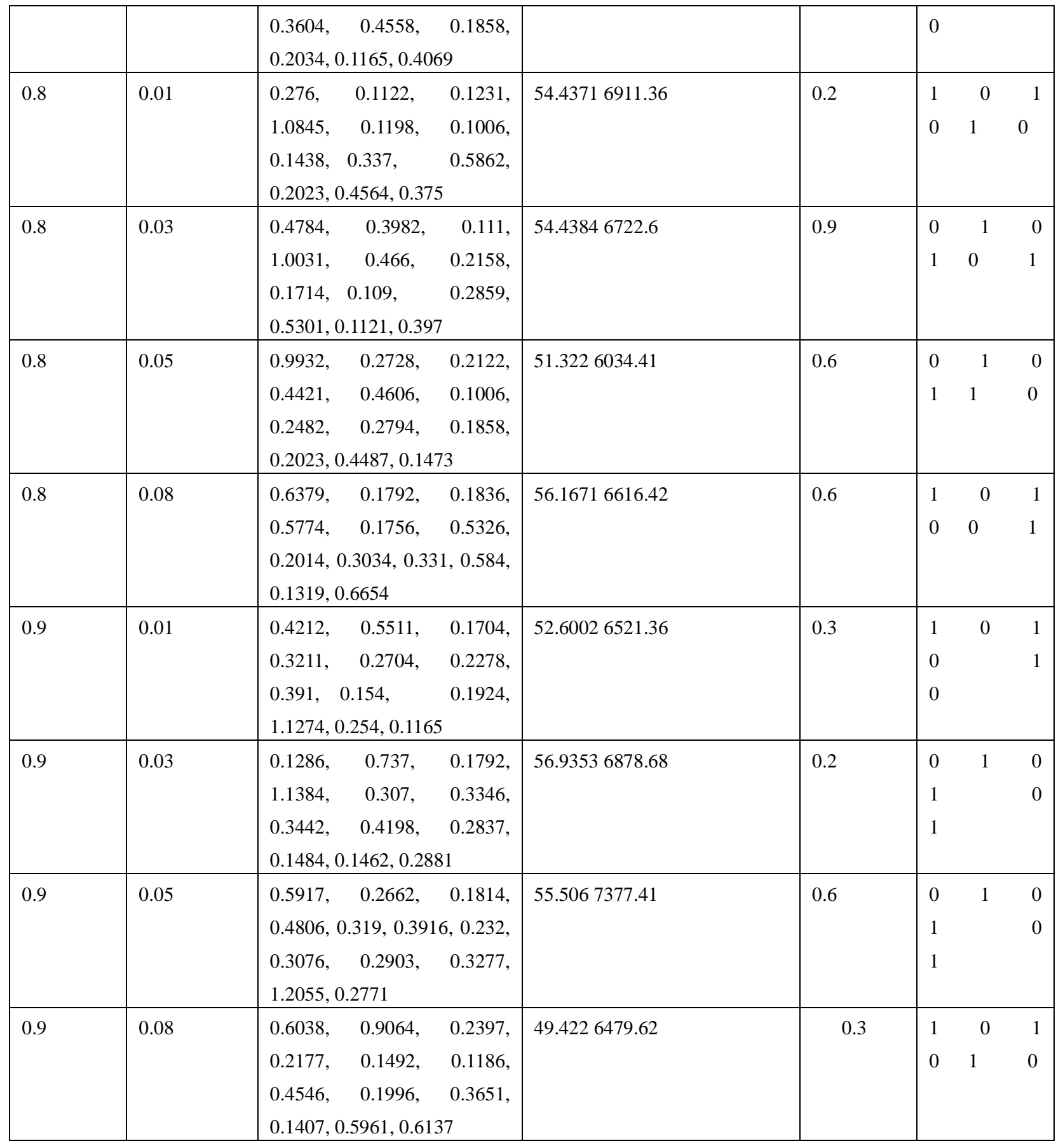

Table 12. $\alpha=0.7$

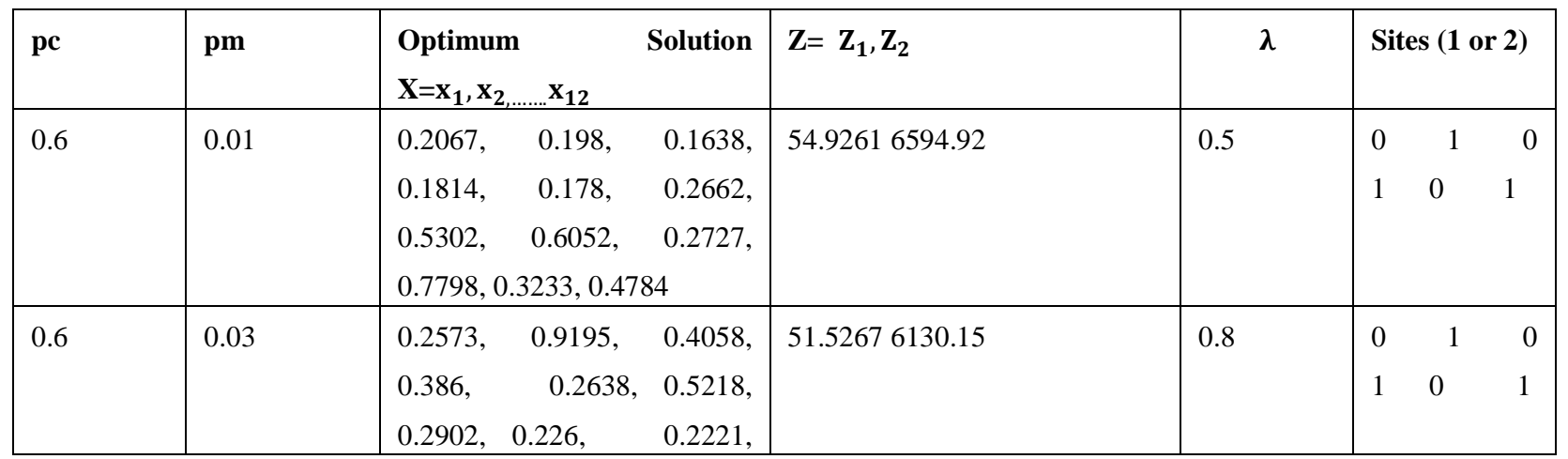




\begin{tabular}{|c|c|c|c|c|c|c|c|}
\hline & & $0.2947,0.2122,0.2606$ & & & & & \\
\hline 0.6 & 0.05 & $\begin{array}{lll}0.694, & 0.2486, & 0.2716, \\
1.1087, & 0.1288, & 0.2752, \\
0.1438, & 0.1426, & 0.1605, \\
0.2925, & 0.3508,0.3651\end{array}$ & 52.15676507 .37 & 0.9 & $\begin{array}{l}1 \\
0\end{array}$ & $\begin{array}{l}0 \\
1\end{array}$ & $\begin{array}{l}1 \\
0\end{array}$ \\
\hline 0.6 & 0.08 & $\begin{array}{l}0.1638, \quad 0.5192, \quad 0.1143, \\
0.3574,0.121,0.4504,0.433, \\
0.3898, \quad 0.3145, \quad 0.1924, \\
0.3662,0.5554\end{array}$ & 47.81455727 .42 & 0.9 & 1 & $\begin{array}{l}1 \\
1\end{array}$ & $\begin{array}{l}0 \\
0\end{array}$ \\
\hline 0.7 & 0.01 & $\begin{array}{lrr}0.727, & 0.3146, & 0.2485, \\
0.1088, & 0.4852, & 0.2458, \\
0.1852, & 0.5506, & 0.2045, \\
0.5708, & 0.7391,0.4058\end{array}$ & 53.81416843 .99 & 0.6 & $\begin{array}{l}1 \\
0\end{array}$ & $\begin{array}{l}0 \\
1\end{array}$ & $\begin{array}{l}1 \\
0\end{array}$ \\
\hline 0.7 & 0.03 & $\begin{array}{lll}0.1308, & 0.6699, & 0.2991, \\
0.4003, & 0.1504, & 0.6106, \\
0.3286, & 0.1204, & 0.1781, \\
0.5983, & 0.1616,0.3783\end{array}$ & 56.19355888 .29 & 0.5 & 0 & $\begin{array}{l}0 \\
1\end{array}$ & 0 \\
\hline 0.7 & 0.05 & $\begin{array}{lll}0.7941, & 0.1364, & 0.4256, \\
0.3981, & 0.5122, & 0.4654, \\
0.2878, & 0.229, & 0.188, \\
0.2683,0.1,0.5708 & \end{array}$ & 53.48796716 .21 & 0.8 & $\begin{array}{l}1 \\
0\end{array}$ & 0 & $\begin{array}{l}1 \\
1\end{array}$ \\
\hline 0.7 & 0.08 & $\begin{array}{lll}0.4971, & 0.5863, & 0.1308, \\
0.5125, & 0.3706, & 0.1756, \\
0.1858, & 0.1654, & 0.5719, \\
0.3365, & 0.7182,0.3706\end{array}$ & 53.9787198 .3 & 0.2 & 1 & ${ }^{1}$ & $\begin{array}{l}0 \\
1\end{array}$ \\
\hline 0.8 & 0.01 & $\begin{array}{lcr}0.2122, & 0.8272, & 0.1297, \\
0.5664, & 0.2218, & 0.214, \\
0.2284, & 0.4786, & 0.1484, \\
0.5356, & 0.2859,0.7545\end{array}$ & 57.03566051 .97 & 0.5 & $\begin{array}{l}1 \\
0\end{array}$ & 0 & $\begin{array}{l}1 \\
1\end{array}$ \\
\hline 0.8 & 0.03 & 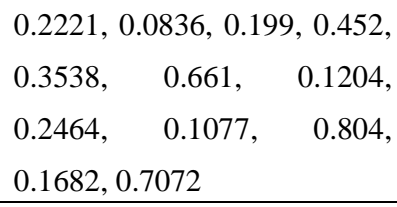 & 53.40975955 .33 & 0.7 & 0 & $\begin{array}{l}0 \\
1\end{array}$ & $\begin{array}{l}1 \\
0\end{array}$ \\
\hline 0.8 & 0.05 & $\begin{array}{lll}0.4949, & 0.8404, & 0.1165, \\
0.4498, & 0.3076, & 0.1246, \\
0.3616, & 0.568, & 0.3398, \\
0.2199, & 0.2419,0.4806\end{array}$ & 52.47146296 .59 & 0.5 & $\begin{array}{l}0 \\
1\end{array}$ & ${ }^{1}$ & $\begin{array}{l}0 \\
1\end{array}$ \\
\hline 0.8 & 0.08 & $\begin{array}{lll}0.5829, & 0.5698, & 0.2958, \\
0.1198, & 0.1738, & 0.4252, \\
0.3382, & 0.4378, & 0.1484, \\
0.3112, & 0.9943,0.6225\end{array}$ & 54.7596779 .52 & 0.1 & 1 & $\begin{array}{l}1 \\
0\end{array}$ & $\begin{array}{l}0 \\
1\end{array}$ \\
\hline 0.9 & 0.01 & $\begin{array}{l}1.1054, \quad 0.3003, \quad 0.2419, \\
0.1539,0.6142,0.187,0.595, \\
0.2158,0.1121, \quad 0.1561, \\
0.3134,0.5499\end{array}$ & 61.65986634 .89 & 0.9 & $\begin{array}{l}0 \\
0\end{array}$ & $\begin{array}{l}1 \\
1\end{array}$ & $\begin{array}{l}1 \\
0\end{array}$ \\
\hline
\end{tabular}




\begin{tabular}{|c|c|c|c|c|c|c|c|c|c|}
\hline 0.9 & 0.03 & $\begin{array}{l}1.115 \\
0.973 \\
0.236 \\
0.261\end{array}$ & $\begin{array}{l}0.480 \\
0.199 \\
0.392 \\
453,0\end{array}$ & $\begin{array}{c}0.122, \\
0.2092, \\
0.2496 \\
8\end{array}$ & 63.82727432 .87 & 0 & $\begin{array}{l}1 \\
0\end{array}$ & $\begin{array}{l}0 \\
1\end{array}$ & $\begin{array}{l}1 \\
0\end{array}$ \\
\hline 0.9 & 0.05 & $\begin{array}{l}0.964 \\
0.679 \\
0.356 \\
0.345\end{array}$ & $\begin{array}{l}0.039 \\
115,0 . \\
0.494 \\
5477\end{array}$ & $\begin{array}{c}0.3662 \\
0.235 \\
0.3112\end{array}$ & 56.87897605 .41 & 0.8 & $\begin{array}{l}1 \\
0\end{array}$ & $\begin{array}{l}0 \\
1\end{array}$ & $\begin{array}{l}1 \\
0\end{array}$ \\
\hline 0.9 & 0.08 & $\begin{array}{l}0.527 \\
0.435 \\
0.434 \\
0.323\end{array}$ & $\begin{array}{c}0.133 \\
0.531 \\
.355, \\
386,0.2\end{array}$ & $\begin{array}{c}0.1187 \\
0.484 \\
0.1407\end{array}$ & 46.75586258 .34 & 0.9 & $\begin{array}{l}0 \\
1 \\
0\end{array}$ & 1 & $\begin{array}{l}0 \\
1\end{array}$ \\
\hline
\end{tabular}

Table 13. $\alpha=0.8$

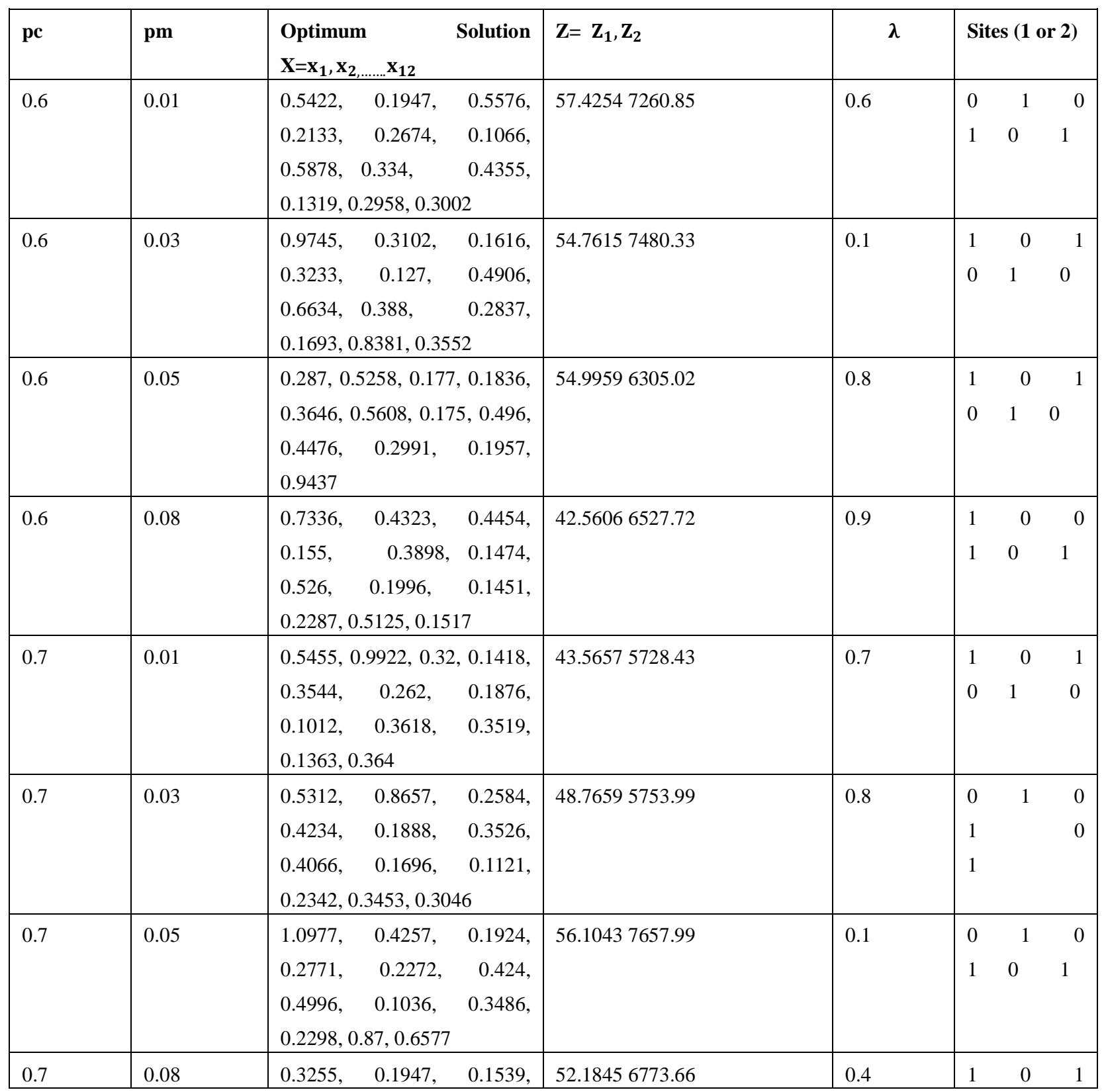




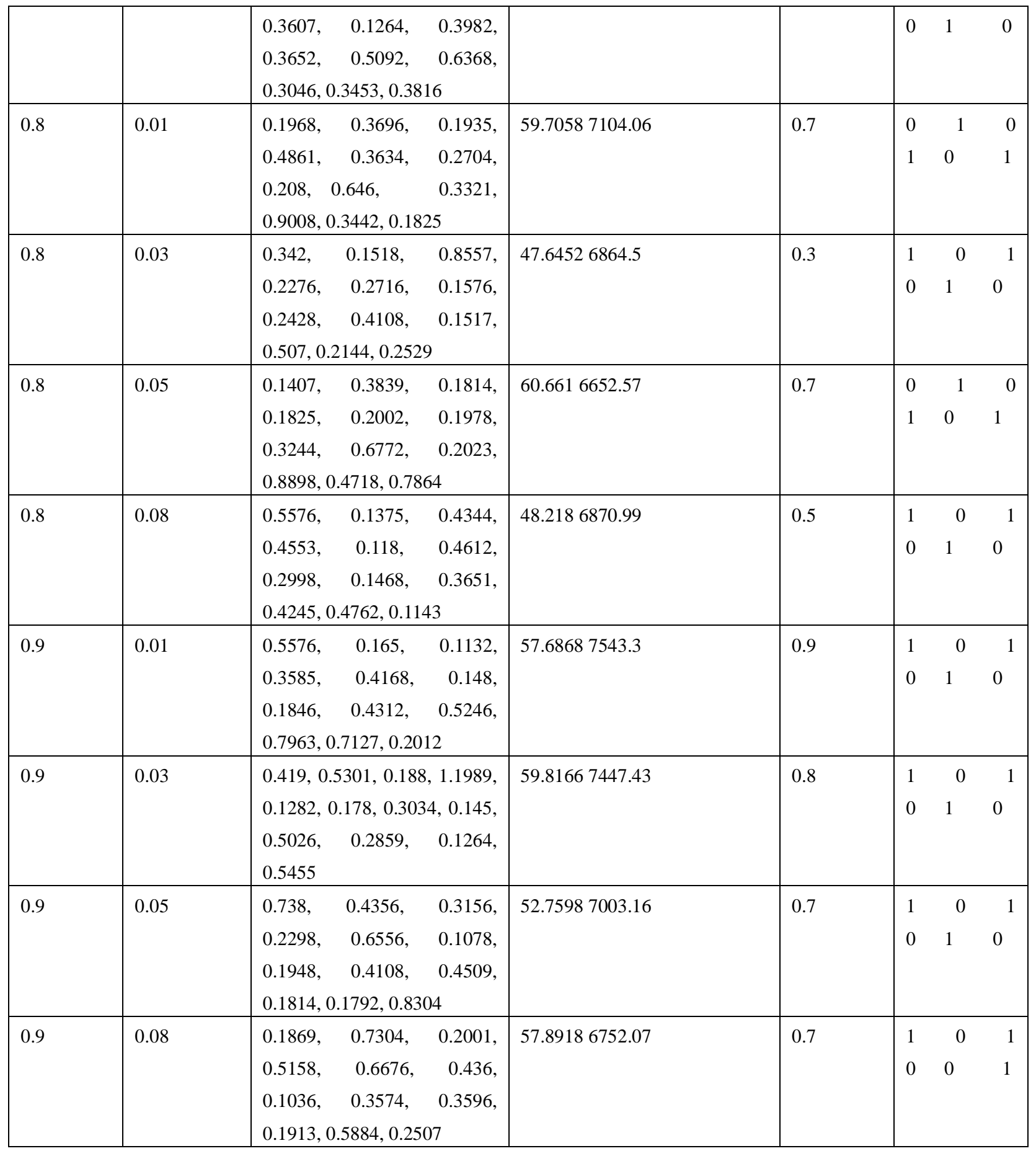

Table 14. $\alpha=0.9$ 


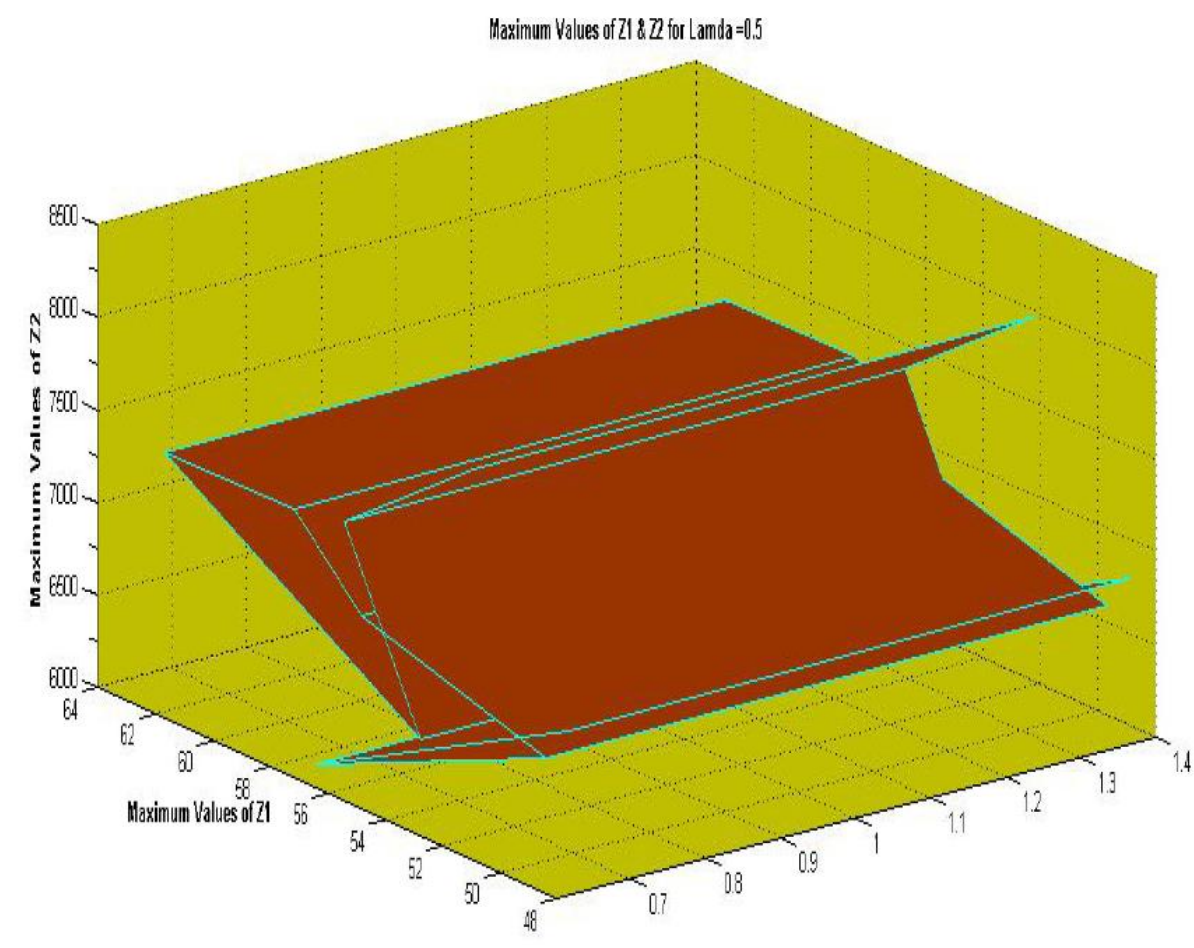

Figure 3. Maximum values of $\mathrm{Z} 1$ and $\mathrm{Z} 2$ for $\lambda=0.5$.

\section{Conclusion and results}

In this article, the multi-objective fuzzy stochastic programming problem for hazardous wastes have been handled using GA based fuzzy programming approach. The bounds of the problem and case study are described as fuzzy Weibull distribution and fuzzy normal distribution, and the confidence levels are treated as fuzzy number. The Table 6-14 show the optimum Pareto's solutions for different values. The Figure $\mathbf{2}$ and $\mathbf{3}$ present the graphical representation of the optimum solutions for $\alpha$ $=0.5$. One of the major advantages of the procedure is that the computational time is greatly reduced to solve the main problem. Another advantage is that it provides the decision maker to have alternative Pareto solutions which readily help them in making appropriate decision regarding the problem. The results of the numerical example and case study showed that the above procedure is very efficient. The number of variables in the problem increases as we moved to some large and complex problems which put the limitation to the proposed methods. The extension of the proposed research model can be made to problems of transportation, transhipment, solid transportation, inventory, etc.

\section{References}

1. Ardjmand E, Weckman G, Park N, et al. Applying genetic algorithm to a new location and routing model of hazardous materials. International Journal of Production Research 2015; 53(3): 916-928.

2. Ardjmand E, Young WA, Weckman GR, et al. Applying genetic algorithm to a new biobjective stochastic model for transportation, location, and allocation of hazardous materials. Expert Systems with Applications 2016; 51: 49-58.

3. Karadimas NV, Kouzas G, Anagnostopoulos I, et al. Urban solid waste collection and routing: The ant colony strategic approach. International Journal of Simulation: Systems, Science \& Technology 2005; 6(12-13): 45-53.

4. Samanlioglu F. A multi-objective mathematical model for the industrial hazardous waste location-routing problem. European Journal of Operational Research 2013; 226(2): 332-340.

5. Current J, Ratick S. A model to assess risk, equity and efficiency in facility location and transportation of hazardous materials. Location Science 1995; 3(3): 187-201.

6. Giannikos I. A multiobjective programming model for locating treatment sites and routing hazardous wastes. European Journal of Operational Research 1998; 104(2): 333-342.

7. List G, Mirchandani P. An integrated network/planar multiobjective model for routing and siting for hazardous materials and wastes. Transportation Science 1991; 25(2): 146-156. 
8. List G, Mirchandani P. Community-focused routing and siting model for hazardous materials and wastes. Proceedings of the National Conference on Hazardous Materials Transportation; ASCE; 1991.

9. Nema AK, Gupta SK. Optimization of regional hazardous waste management systems: An improved formulation. Waste Management 1999; 19(7): 441-451.

10. Gomez JR, Pacheco J, Gonzalo-Orden H. A tabu search method for a bi-objective urban waste collection problem. Computer-Aided Civil and Infrastructure Engineering 2015; 30(1): 36-53.

11. Warmerdam JM, Jacobs TL. Fuzzy set approach to routing and siting hazardous waste operations. Information Sciences-Applications 1994; 2(1): 1-14.

12. Liu B. Minimax chance constrained programming models for fuzzy decision systems. Information Sciences 1998; 112(1): 25-38.

13. Chang NB, Davila E. Siting and routing assessment for solid waste management under uncertainty using the grey mini-max regret criterion. Environmental Management 2006; 38(4): 654-672.

14. Bit AK, Biswal MP, Alam SS. Fuzzy programming approach to multicriteria decision making transportation problem. Fuzzy Sets and Systems 1992; 50(2): 135-141.

15. Hulsurkar S, Biswal MP, Sinha SB. Fuzzy programming approach to multi-objective stochastic linear programming problems. Fuzzy Sets and Systems 1997; 88(2): 173-181.

16. Kumar M, Vrat P, Shankar R. A fuzzy programming approach for vendor selection problem in a supply chain. International Journal of Production Economics 2006; 101(2): 273-285.

17. Liu ST, Kao C. Solving fuzzy transportation problems based on extension principle. European Journal of Operational Research 2004; 153(3): 661-674.

18. Zadeh LA. Fuzzy sets. Information and Control 1965; 8(3): 338-353.

19. Charnes A, Cooper WW. Chance-constrained programming. Management Science 1959; 6(1): 73-79.

20. Mohan C, Nguyen HT. A fuzzifying approach to stochastic programming. Opsearch 1997; 34: 7396.

21. Acharya S, Biswal MP. Solving probabilistic programming problems involving multi-choice parameters. Opsearch 2011; 48(3): 217-235.

22. Sakawa M, Nishizaki I, Katagiri H. Fuzzy stochastic multiob-jective programming. Science \& Business Media 2011; 159.

23. Wang S, Watada J. Fuzzy stochastic optimization: Theory, models and applications. Science \& Business Media 2012.

24. Mousavi SM, Jolai F, Tavakkoli-Moghaddam R. A fuzzy stochastic multi-attribute group decision-making approach for selection problems. Group Decision and Negotiation 2013; 22(2): 207233.

25. Sakawa M, Matsui T. Interactive fuzzy program- ming for stochastic two-level linear programming problems through probability maximization. Artificial Intelligence Research 2013; 2(2): 109-124.

26. Aiche F, Abbas M, Dubois D. Chance-constrained programming with fuzzy stochastic coefficients. Fuzzy Optimization and Decision Making 2013; 12(2): 125-152.

27. Acharya S, Ranarahu N, Dash JK, et al. Solving multi-objective fuzzy probabilistic programming problem. Journal of Intelligent \& Fuzzy Systems: Applications in Engineering and Technology 2014; 26(2): 935-948.

28. Acharya S, Ranarahu N, Dash JK, et al. Computation of a multi-objective fuzzy stochastic transportation problem. International Journal of Fuzzy Computation and Modelling 2014; 1(2): 212-233.

29. Li Y, Liu J, Huang G. A hybrid fuzzy-stochastic programming method for water trading within an agricultural system. Agricultural Systems 2014; 123: 71-83.

30. Holland JH. Adaptation in natural and artificial systems: An introductory analysis with applications to biology, control, and artificial intelligence. United States: University of Michigan Press; 1975.

31. Liu B, Iwamura K. Fuzzy programming with fuzzy decisions and fuzzy simulation-based genetic algorithm. Fuzzy Sets and Systems 2001; 122(2): 253262.

32. Jana RK, Biswal MP. Stochastic simulation-based genetic algorithm for chance constraint programming problems with continuous random variables. International Journal of Computer Mathematics 2004; 81(9): 1069-1076.

33. Jana RK, Biswal MP. Genetic based fuzzy goal programming for multiobjective chance constrained programming problems with continuous random variables. International Journal of Computer Mathematics 2006; 83(2): 171-179.

34. Dutta S, Sahoo BC, Mishra R, et al. Fuzzy stochastic genetic algorithm for obtaining optimum crops pattern and water balance in a farm. Water Resources Management 2016; 30(12): 4097-4123.

35. Dutta S, Acharya S, Mishra R. Genetic algorithm based fuzzy stochastic transportation programming problem with continuous random variables. Opsearch 2016; 53(4): 835-872.

36. Lai YJ, Hwang CL. A new approach to some possibilistic linear programming problems. Fuzzy Sets and Systems 1992; 49(2): 121-133.

37. Buckley JJ. Fuzzy probabilities: New approach and applications. Science \& Business Media 2005; 115.

38. Nanda S, Kar K. Convex fuzzy mappings. Fuzzy Sets and Systems 1992; 48(1): 129-132.

39. Buckley JJ, Eslami E. Uncertain probabilities ii: The continuous case. Soft Computing 2004; 8(3): 193-199.

40. ReVelle C, Cohon J, Shobrys D. Simultaneous siting and routing in the disposal of hazardous wastes. Transportation Science 1991; 25(2): 138-145. 